\title{
PoBery: Possibly-complete Big Data Queries with Probabilistic Data Placement and Scanning
}

\author{
JIE SONG, Northeastern University, China \\ QIANG HE, Swinburne University of Technology, Australia \\ FEIFEI CHEN, Deakin University, Australia \\ YE YUAN and GE YU, Northeastern University, China
}

\begin{abstract}
In big data query processing, there is a trade-off between query accuracy and query efficiency, for example, sampling query approaches trade-off query completeness for efficiency. In this article, we argue that query performance can be significantly improved by slightly losing the possibility of query completeness, that is, the chance that a query is complete. To quantify the possibility, we define a new concept, Probability of query Completeness (hereinafter referred to as PC). For example, If a query is executed 100 times, PC $=0.95$ guarantees that there are no more than 5 incomplete results among 100 results. Leveraging the probabilistic data placement and scanning, we trade off PC for query performance. In the article, we propose PoBery (POssiblycomplete Big data quERY), a method that supports neither complete queries nor incomplete queries, but possibly-complete queries. The experimental results conducted on HiBench prove that PoBery can significantly accelerate queries while ensuring the PC. Specifically, it is guaranteed that the percentage of complete queries is larger than the given PC confidence. Through comparison with state-of-the-art key-value stores, we show that while Drill-based PoBery performs as fast as Drill on complete queries, it is $1.7 \times, 1.1 \times$, and $1.5 \times$ faster on average than Drill, Impala, and Hive, respectively, on possibly-complete queries.
\end{abstract}

CCS Concepts: • Information systems $\rightarrow$ Key-value stores;

Additional Key Words and Phrases: Big data, key-value stores, data query, query completeness, probability, data placement, scanning

\section{ACM Reference format:}

Jie Song, Qiang He, Feifei Chen, Ye Yuan, and Ge Yu. 2021. PoBery: Possibly-complete Big Data Queries with Probabilistic Data Placement and Scanning. ACM/IMS Trans. Data Sci. 2, 3, Article 23 (August 2021), 28 pages. https://doi.org/10.1145/3465375

\section{INTRODUCTION}

Query performance optimization for key-value stores is a critical and challenging task for both industrial and academic practitioners $[1,2]$. Very often, it is too expensive for users to retrieve exact

\footnotetext{
This article is supported by the National Natural Science Foundation of China under grant no. 61672143, and the Natural Science Foundation of Liaoning Province under grant no. 2020-BS-054.

Authors' addresses: J. Song, Y. Yuan, and G. Yu, Northeastern University, 195 Chuangxin Road, hunnan District, Shenyang, Liaoning Province, China, 110167; emails: songjie@mail.neu.edu.cn, yuanye@ise.neu.edu.cn, yuge@mail.neu.edu.cn; Q. He, Swinburne University of Technology, John Street, Hawthorn, Victoria, Australia, 3122; email: qhe@swin.edu.au; F. Chen, Deakin University, 727 Collins Street, Docklands, Victoria, Australia, 3008; email: feifei.chen@deakin.edu.au.

Permission to make digital or hard copies of all or part of this work for personal or classroom use is granted without fee provided that copies are not made or distributed for profit or commercial advantage and that copies bear this notice and the full citation on the first page. Copyrights for components of this work owned by others than ACM must be honored. Abstracting with credit is permitted. To copy otherwise, or republish, to post on servers or to redistribute to lists, requires prior specific permission and/or a fee. Request permissions from permissions@acm.org.

(C) 2021 Association for Computing Machinery.

2577-3224/2021/08-ART23 \$15.00

https://doi.org/10.1145/3465375
} 
answers immediately. Approximate query processing is an alternative to answering users without obvious errors within a short response time [3]. A typical type of approximate queries is sampling queries, which return incomplete query results, or aggregations of incomplete query results. In addition to sampling queries, incomplete queries can also be implemented through approximate query conditions and approximate similarities [4].

Compared with complete queries, incomplete queries trade off query completeness for query efficiency. The weakness of an incomplete query is that there is always an accuracy loss no matter how frequently the queries are executed. For example, when accessing a mobile app to query for preferred restaurants nearby, users are very likely to accept that several restaurants fulfilling their requirements may not be included in the results. In fact, they may not even realize these restaurants are excluded. In such a scenario, it is not worth the long wait for the exact and complete results. However, no matter when, where, and how often users query for preferred restaurants, there is no chance to get them all. In such scenarios, is it worth it to improve "certainly-incomplete" queries to "possibly-complete" queries? The positive answer depends on two guarantees: first, the query performance is far better than the complete query; second, the queries are possibly complete against the certainly incomplete, and such possibilities are also high, say, 0.95 . If the mobile app offers improved queries with the two guarantees, then users could enjoy viewing the preferred restaurants immediately even though some matched restaurants are occasionally not contained in the results.

To satisfy this scenario, this article proposes a possibly-complete query processing approach. Instead of approximating the exact results, it focuses on the possibility of query completeness, that is, the chance that the results contain all the matched records. Such a possibility can be interpreted as "how confident the result set is complete" for a range query or "how confident the result is aggregated from a complete result set" for an aggregated query. In most cases, query performance can be significantly improved at a small loss in the possibility of query completeness. We propose PoBery (POssibly-complete Big data quERY), a "possibly-complete" query approach facilitated by probabilistic data placement and scanning, and its implementation. It accelerates queries by trading off the possibility of query completeness. The major contributions of our study are as follows:

(1) From the aspect of abstract approaches, we propose the idea of "possibly-complete" queries. This is different from existing studies of query relaxation, queries over incomplete data, approximate queries, and queries over uncertain data. We propose data models, probability distribution, data placement algorithms, and data selection algorithms to support the "possibly-complete" queries.

(2) From the aspect of system implementation, we design a system, named PoBery, which supports "possibly-complete" queries. The system architecture, implementation details, and evaluation of PoBery provide references to system developers.

The rest of the article is organized as follows. Section 2 introduces an overview of our idea and solutions with a motivation example, our approach, differences, and challenges. Section 3 defines the logic and physical data models, and the probability model. Section 4 explains how data is probabilistically placed in different blocks. Section 5 describes the cell selection and block selection methods in the scanning. Section 6 briefly introduces the system architecture of PoBery. Section 7 experimentally studies the effectiveness of PoBery, and compares PoBery against three state-of-the-art systems-Drill [5], Impala [6], and Hive on Tez [7, 8]-in terms of their query performance. Section 8 reviews the related work. Section 9 draws conclusions and summarizes future work. 


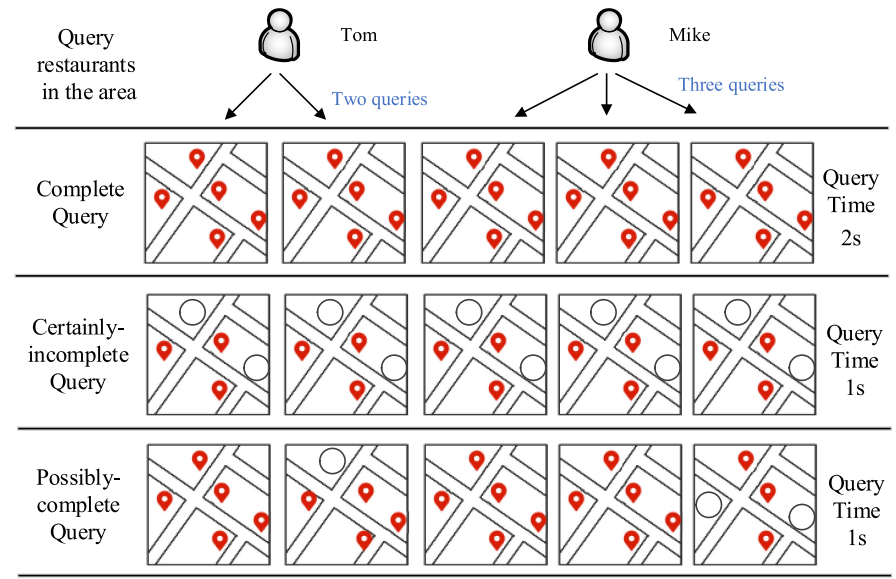

Fig. 1. Example of comparing different query methods.

\section{SOLUTIONS}

This section provides an overview of PoBery. We start our solution with a motivation example. Then, we briefly explain our approach with a figure and discuss the difference between our approach and the sampling-based query, or approximation query, as well as the challenges. In this section, we briefly introduce some new concepts that are formally defined in Sections 3 and 4.

\subsection{Motivation Example}

Suppose the following scenario: Tom and Mike query for preferred restaurants for the same area and conditions through an app, where Tom queries 2 times and Mike queries 3 times (may not be in the same period). Figure 1 shows the results for 5 queries under three different query methods, that is, complete query, certainly-incomplete query, and possibly-complete query as our proposition.

(1) In the complete query, the results of the 5 queries are the same as finding 5 restaurants; each query takes 2 seconds.

(2) In the certainly-incomplete query, the results of the 5 queries are the same as finding the 3 restaurants, and always omitting the 2 restaurants. The query completeness is 0.6 . Due to the fact that about half the results are returned, the query time reduces to 1 second.

(3) In the possibly-complete query, Tom finds 5 restaurants in the first query and 4 restaurants in the second query. Mike's first two queries find 5 restaurants, and the third query finds 3 restaurants. Note that the incomplete results are not the same for different queries. The possibility of query completeness is 0.6 . The completeness of the two incomplete cases is 0.75 , on average. Each query takes 1 second.

The complete query has the best results, but it takes the longest time. Generally, users prefer query efficiency much more than query completeness. They would accept several missing restaurants over a big query delay. The certainly-incomplete query trades off query completeness for query efficiency-it takes less time. However, for users, it is unacceptable that every query is incomplete and the two restaurants are always missing. The possibly-complete query trades off the possibility of query completeness for query efficiency. It takes a short time, has a chance to get complete results, and has the randomness of incomplete query results. 


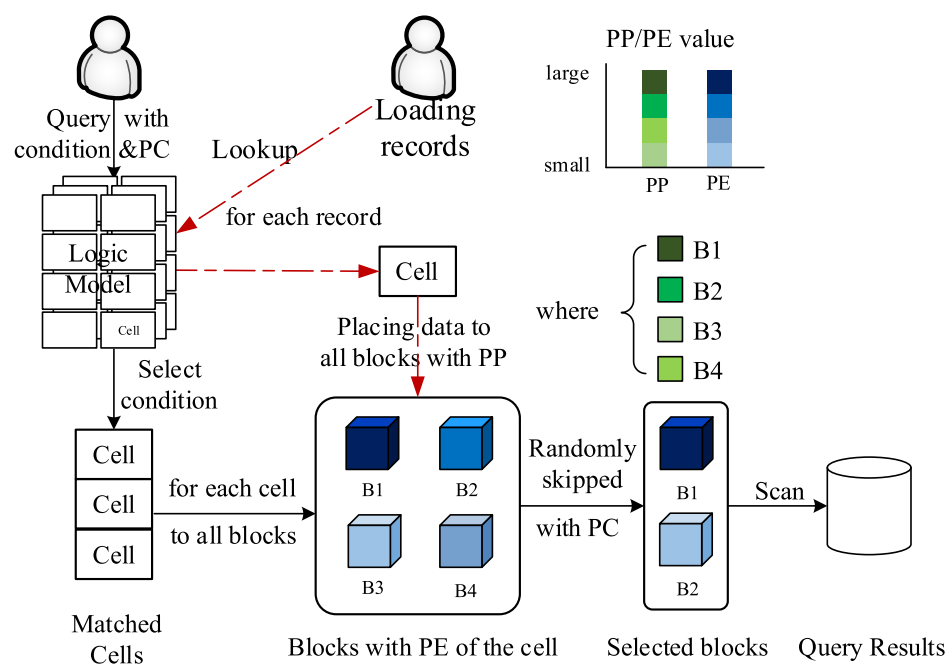

Fig. 2. Sketch of PoBery.

\subsection{Our Approach}

PoBery employs the Probability of query Completeness (PC) to measure the possibility of query completeness and trades off PC for query performance. PC is the probability that the query results are complete or aggregated from a complete result set. To carry out possibly-complete queries, the raw data in the key-value store is first placed as blocks according to a predefined Probability of Placement (PP). Then, through data accumulation, the probability of data stored on a block, referred to as Probability of Existence (PE), is calculated. Finally, the scanner selectively scans these blocks to fulfill the PC while trading off the PC for query performance.

Figure 2 shows the five core concepts employed by PoBery: a cell is a logical data container; a block is a physical data container. Given a cell and a block, PP is the probability that PoBery places each record in the cell to the block, and PE is the probability that the block stores any records of the cell after many rounds of data placement; confidence is a PC guarantee. Given a query condition with confidence, PoBery inspects the PEs of matched cells, selectively scans the blocks, improves query performance, and ensures that the PC is higher than the confidence. For example, if confidence $=1$, all of the blocks will be scanned. If confidence $=0.5$, fewer blocks will be scanned. Here, the confidence is not the sampling rate. Confidence $=0.5$ does not mean that exactly $50 \%$ of blocks will be scanned. PoBery does not guarantee how many blocks are skipped because the PE distribution is not uniform. In contrast, PoBery guarantees that there are fewer than $50 \%$ incomplete results if the query is executed many times.

\subsection{Differences and Challenges}

Unlike the sampling-based query or approximation query, PoBery has two characteristics. First, PoBery focuses on the probabilities of data placement, the approximation query focuses on the similarities of data comparison, and the sampling-based query focuses on the sampling methods of data selection. They leverage different techniques. Second, let query accuracy be the similarity between the query results and the true answers. Then, the approximation query and sampling-based query are inaccurate queries whose accuracy can be measured. While PoBery supports the possibly inaccurate query, both the possibility and accuracy can be measured. Diversely, the optimization effects of PoBery, the sampling-based query, and the approximation query are dominated by the PC 
loss, the sampling rate, and the query accuracy, respectively. PoBery is based on the probabilistic data placement because the deterministic one cannot satisfy our scenario mentioned in Section 1. The sampling queries and incomplete queries can be easily supported by the deterministic data placement. However, their query completenesses are always deterministic if the data placement is known. In addition, PoBery's probabilistic data placement strategy is quite lightweight. It does not incur extra storage costs because it does not rely on any pre-computation and look-up structures tables. It does not require any hash calculation on data because it is value independent. The mapping between values and data blocks is solved in the logical data model, but not in the data placement. To this end, PoBery addresses the following issues as the main challenges of this study.

(1) How to quantitatively model the relationships between query performance, PC, PE, and $\mathrm{PP}$

(2) How to ensure both the non-uniform probabilities of the data stored on a block for probabilistic data placement and, to the contrary, the uniform sizes of blocks for data balance. The data matching the query conditions have different PEs on different data blocks due to the non-uniform PE distribution. Thus, the sizes of different data blocks are imbalanced. For all of the keys, the challenge is how to manage the balance between data blocks with a predefined data arrangement.

(3) How to ensure both the PC requirement and the randomness. The latter requires that the data placement algorithm must select blocks randomly if a query is executed many times with the same confidence. Without the randomness, two unexpected consequences may occur when confidence $\neq 1$. First, if a block with a small PE actually contains matched data, it will always be ignored, and the query results will always be incomplete. Second, incomplete result sets will always be the same.

\section{MODELS}

\subsection{Data Model}

PoBery's data model consists of a logical part and a physical part. It follows the well-known keyvalues data model used in most key-value stores, where a table contains many records and each record contains many key-value pairs. In this article, we give "key" an alias as "attribute." In the following discussion, we focus on how PoBery processes an individual table with many attributes (keys). Given multiple tables, PoBery reuses the solution of a single table to process them individually. PoBery's logical data model is a tree-structured tablespace whose levels are frequently-queried attributes in the table. Records are equal-frequently partitioned level by level, and to the bottom. The leaf nodes of a tablespace, named as cells, contain the same number of records. PoBery's physical data model includes blocks and trunk files, which store the records.

Definition 1 (Query Attribute and Segment). In a table, the query attributes are attributes that have three features: they are non-empty attributes (or have a default value); they are independent of each other; and they frequently occur in the query conditions. Let $\left\{d_{1}, d_{2}, d_{3}, \ldots, d_{z}\right\}$ be the $z$ query attributes sorted by their frequency, that is, $d_{i}$ is more frequent as a query condition than $d_{j}$ if $1 \leq$ $i<j \leq z$. A segment represents a range of values, namely, it is a two-tuple $<\alpha, \beta>$ representing the lower boundary and upper boundary of the range. A query attribute is divided into many segments and each segment is a container of records. Let $s$ be a segment of a query attribute $d$ and a record whose value on $d$ is $u$; then, $s$ contains the record iff $u \in(a, \beta]$ when $u$ is a numerical value, or dictionary $(u) \in(a, \beta]$ when $u$ is textual value. Function dictionary() is the order of $u$ 's 5 -character prefix in all 5-character permutations; therefore, it is easy to support the precise queries and prefix queries on textual data. 


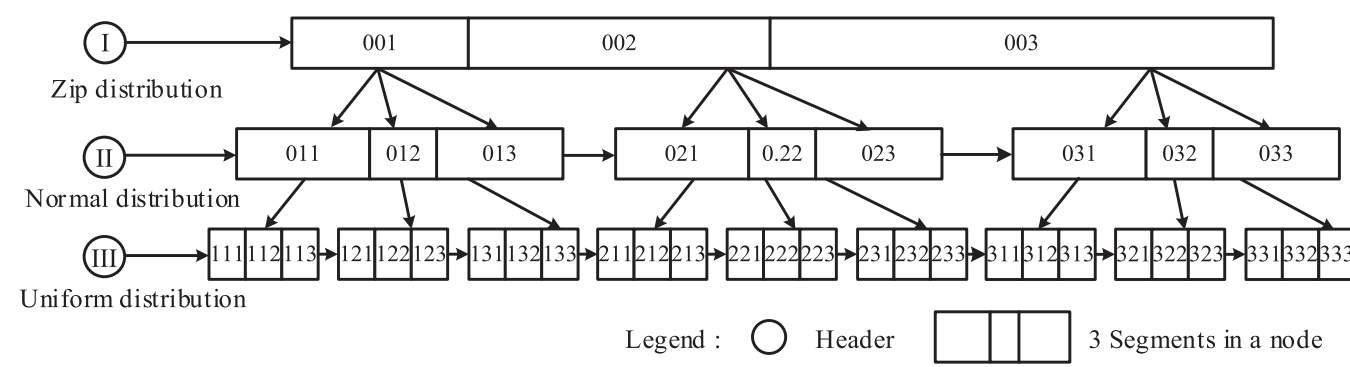

Fig. 3. An example of tablespace with 3 query attributes whose data distributions are different.

Definition 2 (Cells). A cell is a logical container of records. It consists of a segment on each query attribute. Let $s_{i}$ be an arbitrary segment of query attribute $d_{i}$; then, $\left.<s_{1}, s_{2}, s_{3}, \ldots, s_{z}\right\rangle$ is a cell iff it satisfies two rules:

(1) Cells are non-overlapping-a record is contained by one and only one cell;

(2) Cells are equal-frequency-the number of records in each cell is the same.

Given a table, the number of cells is predefined. Records are partitioned into these cells in equal sizes.

Definition 3 (Tablespace and Levels). Given a table, its tablespace is a data structure that represents how the segments are linked and organized to cells. Let a table have $z$ query attributes, its tablespace is a " $\kappa$-ary," full, and ordered tree with links of siblings and head-node of each level. In the tablespace, the levels are query attributes ordered from top to bottom, and the $i$-th $(1 \leq i \leq$ $z$ ) level has a $\kappa^{(i-1)}$ node and each node has $\kappa$ segments. Based on the tablespace, the records are first partitioned into $\kappa$ equal-frequency and non-overlapping segments of $d_{1}$; after that, records in each $d_{1}$ 's segment are further partitioned into $\kappa$ equal-frequency and non-overlapping segments of $d_{2}$, and then this operation continues until $d_{z}$ is reached. Finally, there are $\kappa^{(z-1)}$ leaf nodes and each leaf node contains $\kappa$ segments so that there are $\kappa^{z}$ cells. The range of a cell in a leaf node is the union of the ranges of segments, such as $\left\langle s_{1}, s_{2}, s_{3}, \ldots, s_{z}\right\rangle$, on the path of the root to the leaf node.

Figure 3 shows a tablespace where $z=3$ and $\kappa=3$. The tablespace creation algorithm is intuitive. Its key step is equal-frequency binning that sorts the records first and then partitions them by data amount [9]. The binning is known as $\mathrm{O}(n \log n)$ algorithm, where $n$ is the number of records. The details are abbreviated in this article; see also the experiment in Section 7.3. In Figure 3, the data distributions of three independent query attributes are different, and the length of boxes at the same level means the range size of the segments. The head node of each level and links between siblings are for traverse because a query condition may not contain every query attribute (see also Algorithm 1 in Section 5.1).

Definition 4 (Trunks and Blocks). Trunks, or trunk files, are fixed-size files in a distributed file system. Blocks are the containers of these trunks. They are implemented as directories on a distributed file system.

To summarize, cells are the logic containers of records. Records are categorized into cells. Trunks, which are files maintained by a host file system, are the storage of records. Blocks, which are the physical locations, are the containers of trunks. The number of cells dominates the number of blocks, and the data volume dominates the number of trunks in a block, called blocks size. Data balancing ensures that the sizes of different blocks are almost equivalent, as discussed in Section 4 . 


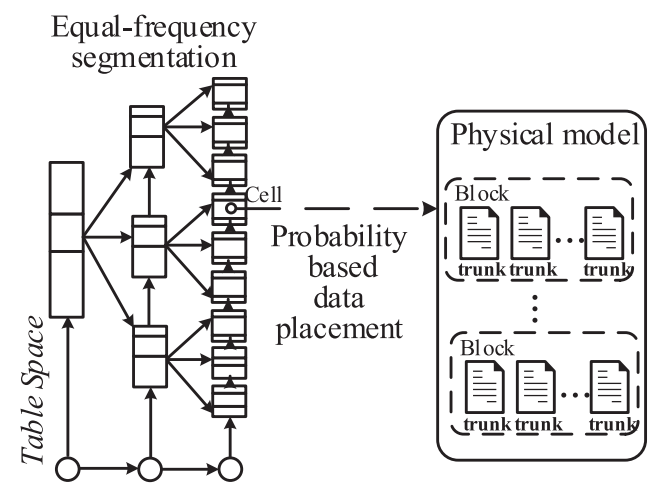

Fig. 4. The relationship among logical data model, block, and physical data model.

Figure 4 illustrates PoBery's data model. For the logical data model, the tree-structure tablespace has three levels: $d_{1}, d_{2}$, and $d_{3}$. The segments of these levels partition the space into many cells. The numbers of records in different cells are almost the same. For the physical data model, there are many trunks in each block. PoBery maintains the mapping relationships among records, cells, blocks, trunks, and nodes (machines) as follows.

(1) Records to cells: Many-to-one relationships are maintained using a tablespace.

(2) Records to blocks: One-to-one relationships are implemented via probabilistic data placing. A record may be placed in any block but will finally be placed in one block.

(3) Cells to blocks: Many-to-many relationships are defined as an "existence," that is, a cell exists in a block if any record in the cell is placed in the block.

(4) Trunks to blocks: Many-to-one relationships are maintained by PoBery, similar to the relationship between files and folders. More trunks are "mapped" to a block if more records are placed in the block.

(5) Blocks to nodes: Many-to-many relationships are implemented by distributing the trunks of a block to all nodes uniformly.

(6) Trunks to nodes: Many-to-one relationships are maintained by a distributed file system.

The probability model, which is adopted to build (2) and calculated point (3), will be described next.

\subsection{Probability Model}

PoBery's probability model consists of PC, PE, and PP. PC is deduced by PE, that is, the probability that a cell exists in a block. PE is the accumulative probability of PP, that is, the probability that a record is placed in a block.

Definition 5 (Probability of Query Completeness (PC)). The PC is the probability that a query result is complete or is aggregated from a complete result set. It indicates what the confidence is that a query result set is complete.

PC is not a measure of query completeness. For instance, when a result set contains $90 \%$ of true results, the query completeness is 0.9 . However, let the PC of a query be 0.9 , and it measures that if the query is executed many times, $90 \%$ of cases whose query completeness are 1 and $10 \%$ of cases whose query completeness are not 1 , but rather any values between 0 and 1 .

Definition 6 (Probability of Placement $(P P)$ ). Given a record in a cell $c_{i}$ and a block $b_{j}$, the probability that the record is placed (stored) in $b_{j}$ is defined as its probability of placement, denoted as $p_{i j}$. 
Let $m$ be the number of cells and $n$ be the number of blocks. PP is represented as an $m \times n$ matrix, where $p_{i j}=f_{D P A}(i, j)$. Function $f_{D P A}(i, j)$ is predefined, and the data placement is executed periodically based on the matrix. This operation will be discussed in detail in Section 4.

Definition 7 (Probability of Existence (PE)). Given a cell $c_{i}$ of a tablespace and a block $b_{j}$, the probability that $b_{j}$ contains the records of $c_{i}$ is defined as the probability of existence. PE is associated with a cell and a block because the cell exists in the block if any record in the cell is placed in the block. Let $e_{i j}$ denote the PE of $c_{i}$ to $b_{j}$. On the contrary, the Probability of Not Existence, denoted as PNE, equals 1-PE.

$\mathrm{PE}$ is represented as an $m \times n$ matrix, where element $e_{i j}$ is the probability of $c_{i}$ existing in $b_{j}$. $c_{i}$ with $\omega$ records means that $c_{i}$ has been processed by data placement for $\omega$ times and for each time one record is placed. Therefore, the PNE of $c_{i}$ to $b_{j}$ is $\left(1-p_{i j}\right)^{\omega}$, and there is $e_{i j}=1-\left(1-p_{i j}\right)^{\omega}$.

\section{DATA PLACEMENT}

Data placement is the method of placing the records of cells into trunks in blocks. PC-based querying relies on data placement. In this section, PoBery's data placement and PP distribution algorithms are discussed.

\subsection{One Cell}

In this section, we explain how the records in one cell are placed according to the PP distribution for the cell. The PP distribution is how PPs are distributed among all blocks, denoted as $f(x)$.

Function $\boldsymbol{h}(\boldsymbol{x})$. Let a continuous function $h(x)$ satisfy:

(1) $h(x) \in(0,1)$;

(2) $\int_{0}^{\lambda} h(x) d x=1-\varepsilon$, where $\lambda$ is a selected positive number, and $\varepsilon$ is a very small value.

Then, given a cell, $h(x)$ is modeled as the probability density function of the cell. $H(x)$ is the primary function of $h(x)$.

Function $f(x)$. Given a cell, $f(x)$ is its PP to the block $x$. Let $n$ be the number of blocks, where $n>\lambda, n \% \lambda=0, \Delta=n / \lambda$. PoBery numbers the blocks from 1 to $n$. The PP of the block $a$ is $\int_{(a-1) \Delta}^{a \Delta} h(x) d x=H(\Delta a)-H(\Delta(a-1))$. Define $f(x)=H(\Delta x)-H(\Delta(x-1))$. When a record of the cell is loaded, PoBery randomly generates a seed in the range of $f(x)$. Then, the record is placed in the block $\left\lfloor f^{1}(\right.$ seed $\left.)\right\rfloor$, or randomly select one of them if they are not unique.

Function $g(x)$ : Given a cell, $g(x)$ is its PE to block $x$. If there are $\omega$ records, after data placement, the PE of the cell on block $x$, denoted as $g(x)$, is $1-(1-f(x))^{\omega}$.

The definition of $g(x)$ is inspired by Amdahl's Law [10]. It predicts the theoretical speedup when using multiple processors. Compared with a parallelable task running in a stand-alone environment, when a few processors are introduced, the speedup is obvious. However, when the number of processors increases, the speedup tends to stabilize. PoBery is designed in a similar manner. In PoBery, PE is the dominant attribute of probabilistic queries. We then design so that the PEs of blocks satisfy the rule "more small values and less large values." Therefore, the speedup is obvious when PC slightly decreases because many blocks with small PEs are skipped in the data scanning, while speedup tends to stabilize when PC greatly decreases because fewer blocks with larger PE are skipped in the data scanning. We select the normal distribution as $h(x)$ and deduce the functional image of $g(x)$, as shown in Figure 5.

$$
\begin{gathered}
h(x)=N(u, \sigma 2)=\frac{1}{\sqrt{2 \pi \sigma^{2}}} e^{-\frac{(x-\mu)^{2}}{2 \sigma^{2}}} \\
H(x)=\frac{1}{2}\left[1+\operatorname{erf}\left(\frac{x-\mu}{\sigma \sqrt{2}}\right)\right]
\end{gathered}
$$



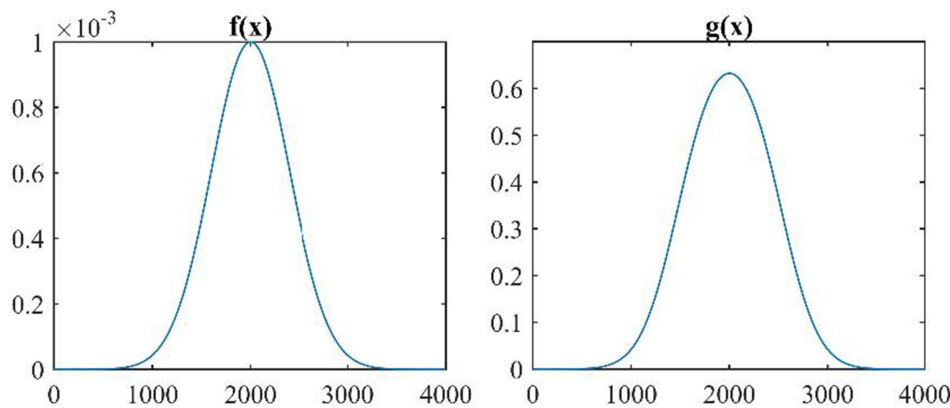

Fig. 5. Examples of $f(x)$ and $g(x)$ when $\omega=1,000, \lambda=4, n=4,000, \sigma=0.3989, \mu=0.5 \lambda$.

Table 1. Example of Data Placement When $m=3$ and $n=6$

\begin{tabular}{l|ccc}
\hline & Cell 1 & Cell 2 & Cell 3 \\
\hline Block 1 & $F_{D P A}(1,1)=f(1)$ & $F_{D P A}(2,1)=f(3)$ & $F_{D P A}(3,1)=f(5)$ \\
Block 2 & $F_{D P A}(1,2)=f(2)$ & $F_{D P A}(2,2)=f(4)$ & $F_{D P A}(3,2)=f(6)$ \\
Block 3 & $F_{D P A}(1,3)=f(3)$ & $F_{D P A}(2,3)=f(5)$ & $F_{D P A}(3,3)=f(1)$ \\
Block 4 & $F_{D P A}(1,4)=f(4)$ & $F_{D P A}(2,4)=f(6)$ & $F_{D P A}(3,4)=f(2)$ \\
Block 5 & $F_{D P A}(1,5)=f(5)$ & $F_{D P A}(2,5)=f(1)$ & $F_{D P A}(3,5)=f(3)$ \\
Block 6 & $F_{D P A}(1,6)=f(6)$ & $F_{D P A}(2,6)=f(2)$ & $F_{D P A}(3,6)=f(4)$ \\
\hline
\end{tabular}

\subsection{Multiple Cells}

In this section, we extend the data placement of one cell to multiple cells. If $f(x)$ is applied to all cells directly, a block has the same PP for the records in all of the cells. In such a case, the data distribution among blocks, which is in accord with $g(x)$, is imbalanced. Blocks are logic containers of trunks, and trunks of a block are uniformly stored among physical nodes. Therefore, we prefer a balanced data distribution among blocks because it allows parallelism.

Definition 8 (Data Placement Algorithm $(D P A)$ ). The data placement algorithm is a function $F_{D P A}$ : $(i, j) \rightarrow p_{i j}$, where $i \in[1, m]$ is the index of a cell in a tablespace, $j \in[1, n]$ is the index of a block in the physical model, and $p_{i j}$ is the probability of placement. The DPA satisfies the following three conditions.

(1) $\forall i \in[1, m], F_{D P A}(i, 1)+F_{D P A}(i, 2)+\cdots+F_{D P A}(i, n)=1$. That is, a record has to be placed in a block.

(2) $\forall i \in[1, m], j \in[1, n], F_{D P A}(i, j) \neq 0$. That is, given a record, each block has a chance to get this record.

(3) $\forall a \in[1, m], F_{D P A}(a, j)$ is a continuous function with less large values and more small values. That is, the data distribution of blocks is imbalanced for one cell.

(4) $\left.\forall b_{1} \in[1, n], b_{2} \in 1, n\right], b_{1} \neq b_{2} \sum_{i=1}^{m} F_{D P A}\left(i, b_{1}\right)=\sum_{i=1}^{m} F_{D P A}\left(i, b_{2}\right)$. That is, the data distribution of blocks is balanced for all of the cells.

Function $f(x)$ matches conditions (1) to (3) for one cell, and an offset function offset $(i, j)$ is adopted for condition (4). Let the offset be $\delta=n / m$, and

(1) $x=\operatorname{offset}(i, j)=j+(i-1) \delta$ when $j+(i-1) \delta \leq n$;

(2) $x=\operatorname{offset}(i, j)=j+(i-1) \delta$ - $n$ when $j+(i-1) \delta>n$.

Then, there is $F_{D P A}(i, j)=f[o f f s e t(i, j)]$. For example, if $m=3, n=6, \delta=2$, the $F_{D P A}$ and $f$ functions are mapped as shown in Table 1. With the offset, the accumulative PPs for all of the cells on any 


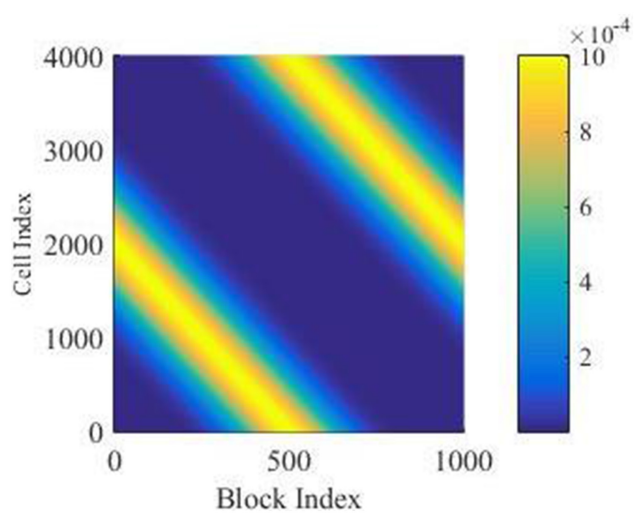

Fig. 6. PPs for cells to blocks.

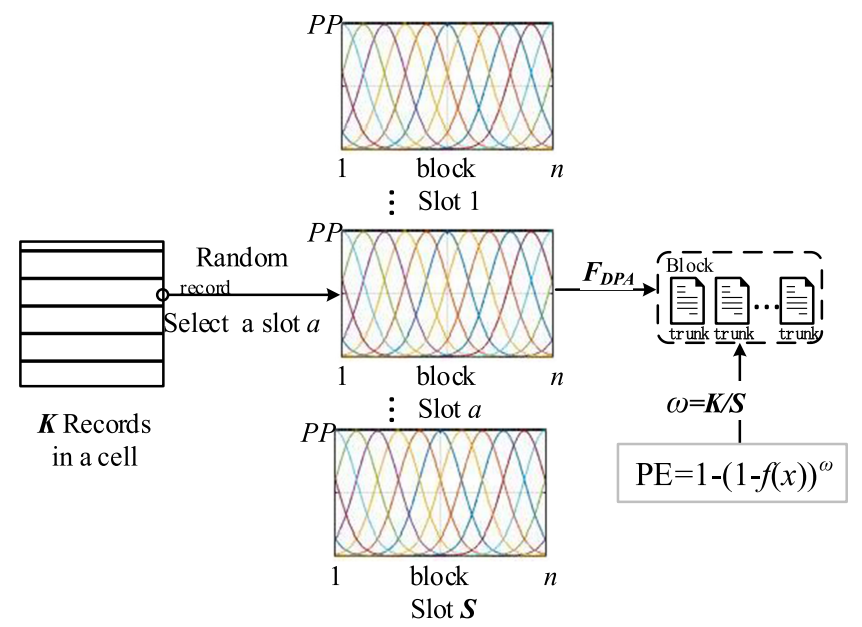

Fig. 7. Slots in data placement.

block are the same. For example, the value is 0.25 in the case of $m=1000, n=4000, \omega=1000$, $\lambda=4, \sigma=0.3989, \mu=0.5 \lambda$. Figure 6 shows the PPs in gradient colors for all of the cells to all of the blocks under these settings. The symmetry of the blocks is caused by the offset. Figure 6 proves both conditions (3) and (4) in Definition 8.

\subsection{Practice Experiences}

In this section, we introduce the practice experiences for improving the efficiency of the proposed data placement. To avoid the situation where PE is either 0 or 1 for most blocks due to the extremely large $\omega$ value, we introduce the concept of slot. Slots are containers of blocks. If the blocks are folders, slots are parent folders. Each slot contains $n$ blocks. The PP and PE distributions of blocks in each slot are independent and identical, that is, a block $b_{j}$ is contained in every slot and they are identical. As shown in Figure 7, when a record in a cell is placed, the record randomly selects a slot as a destination. Then, $F_{D A P}$ is performed in the slot. Thus, the $\omega$ value is the number of records in a cell divided by the number of slots.

Data placement in a block is straightforward. As discussed in Section 3, a block may contain records from all of the cells, and trunks are the files for storing these records. To reduce the scan scope, the trunks in a block must distinguish the records from different cells. However, this strategy 


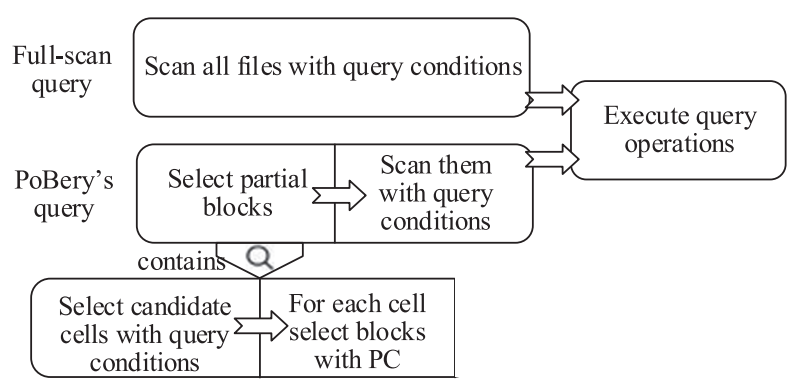

Fig. 8. Comparison between the full-scan query and PoBery's query.

is impractical because the cells are numerous. In addition, trunks are numerous and most are small files if a trunk stores the records in only one cell. PoBery adopts another strategy to place records among trunks. First, the trunks of a block are distributed uniformly among nodes to ensure parallelism. Second, records are sequentially written to trunks row by row where the cell index is the identifier of each row stored as its header. Therefore, during the query processing, trunks are scanned in parallel and only the headers of rows are scanned to determine whether the rows belong to the queried cells.

In practice, PoBery conducts the data placement in terms of neither cell granularity nor record granularity, but rather in terms of batch granularity. A batch is a small set of records that belong to the same cell. Batch size is dominated by the cache size during data loading. Records are first loaded to the write buffer and then grouped into batches by their cells. Finally, they are placed to blocks batch by batch. The batch size is not fixed and does not affect the probability model. This method also reduces the placing frequency, avoiding the extremely large $\omega$. Some implementation details of the data placement algorithm, such as load balancing, can be found in our previous work [11].

\section{SCANNING}

This section presents the scan operation. We first explain how the scan works in a PoBery query operation. Figure 8 shows the comparison between the full-scan query and PoBery's query. The full-scan query process scans data files of all blocks with query conditions and then executes specific query operations, such as aggregation, join, sort, and so on. In contrast, PoBery changes the first step of the query process. It selects and scans partial blocks, which possibly contain the results, instead of all blocks. Then, it scans the trunks in the partial blocks to retrieve the results that satisfy the query conditions, taking the union of the results in these blocks as scan results. In PoBery, the updated operation contains two steps: cell selection and block selection.

\subsection{Cell Selection}

Cell selection is the operation that selects the candidate cells from the tablespace according to the query conditions. Candidate cells are the minimal set of cells containing the query results. Given query conditions $Q$, after the cell selection operation, the corresponding levels and segments in the tablespace are located and the corresponding cells are selected. Note that some levels may not in $Q$, and one or more segments in a level may joint with $Q$. Algorithm 1 shows the cell selection algorithm.

The cell selection algorithm includes the entrance step and recursive step. The former finds the first level that is in the query conditions by top-down traverse, and treats the head node of the level as the entrance of selection. The latter selects the match segments from this level, and all match 


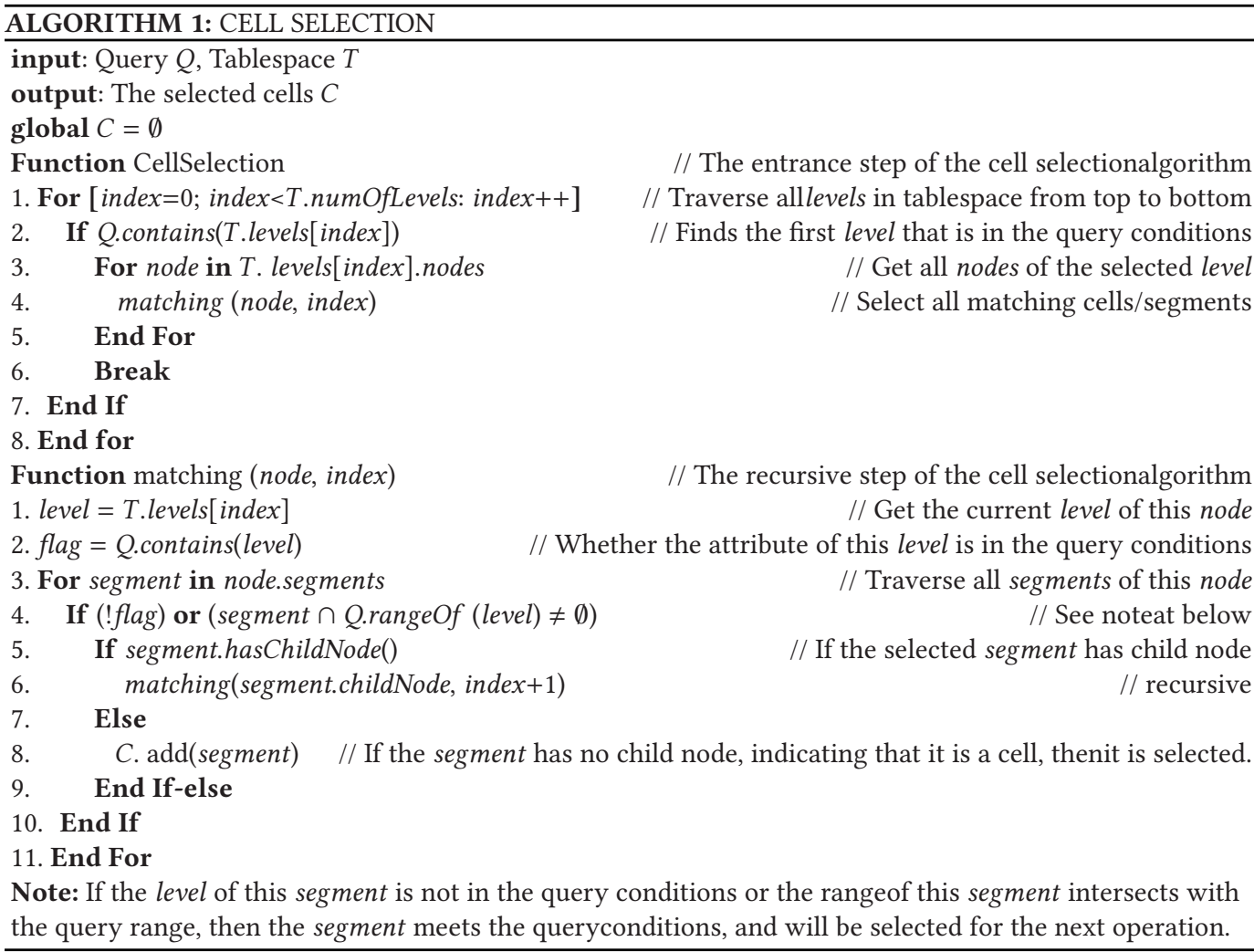

subsegments recursively. In the recursive step, the segments in a level are all matched if the level does not occur in the query conditions. Let a tablespace have $z$ levels and a node have $\kappa$ segments; the algorithm is a variant of depth-first traversal on a full $\kappa$-ary tree. The time complexity is $\mathrm{O}\left(z^{\kappa}\right)$ due to the fact that the best situation is $z^{\kappa}$ and the worst situation is $z^{1}+z^{2}+\cdots+z^{\kappa}=z\left(z^{\kappa}-1\right) /$ $(z-1)$.

The cell section cannot filter any blocks because the records in a cell could be stored in any blocks. For this reason, the cell section is not an indexing mechanism, and no mappings between cells and blocks are maintained. For each cell in the candidate set, every block is examined with the PE of the cell and the block.

\subsection{Block Selection}

Block selection is next to cell selection. For each cell, blocks are selected using a heuristic blockselection algorithm (H-selection) whose stop mechanism is the PC exceeding the confidence.

Heuristic block-selection (H-selection). $\mathrm{H}$-selection takes matched cells $\left\{c_{i}\right\}$ and confidence $p_{0}$ as the inputs, and outputs the selected blocks $\left\{b_{j}\right\}$. It ensures query randomization by selecting blocks randomly. The query results are different when the same query is executed multiple times with the same confidence. H-selection ensures that the blocks with a small PE can be queried instead of being skipped every time. Therefore, when blocks are selected, both PE and randomization are considered. The pseudo-code of H-selection is shown as Algorithm 2, whose time complexity is $\mathrm{O}(\mathrm{n})$, where $n$ is the number of blocks. 


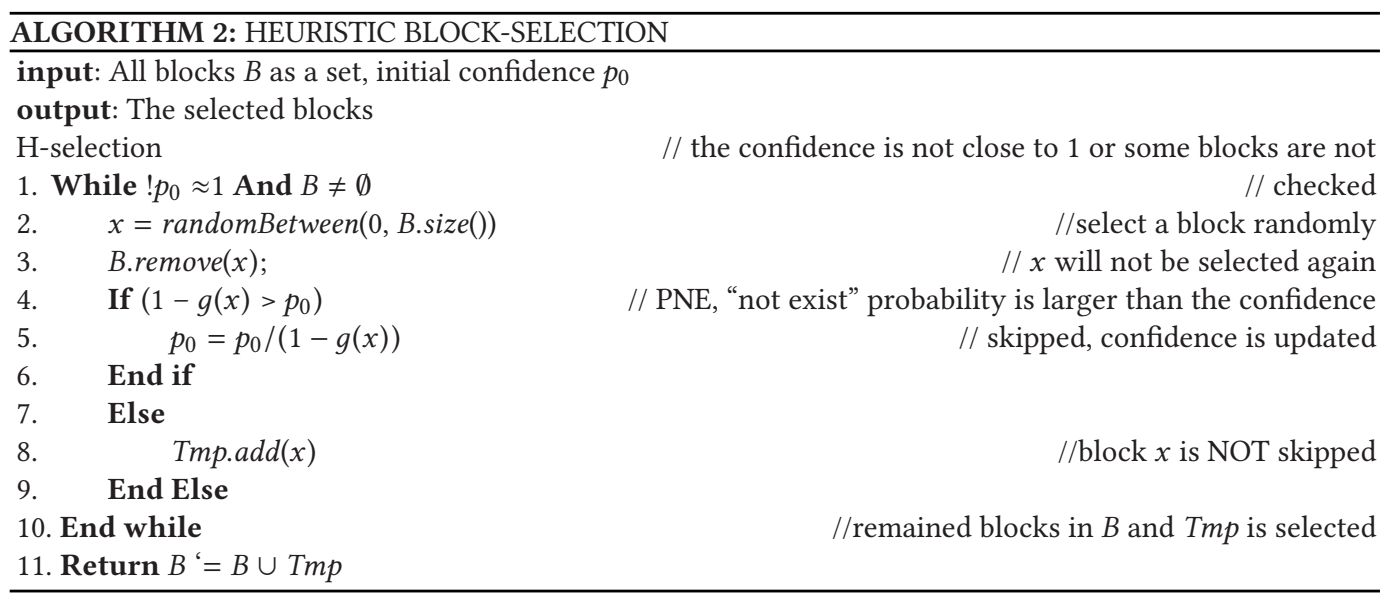

For concision and without loss of generality, let the first cell be the query target and let block $x$, randomly selected from all blocks $B$, be the first block for consideration under the confidence $p_{0}$. Then, the PE of block $x$ is $g(x)$, and its PNE, denoted as $p n e_{x}$, is $1-g(x)$. If $p n e_{x}>p_{0}$, it indicates that the possibility of "records of the cell being in other blocks" is already higher than the required confidence. Consequently, block $x$ is skipped because scanning it does not increase the PC. Meanwhile, the confidence is updated to $p_{0} /$ pne $_{x}$ because $\mathrm{P}(B \mid B-x)=\mathrm{P}(B \cup B-x) / \mathrm{P}(B-x)=\mathrm{P}(B) / P N E x=$ $p_{0} /(1-g(x))$, where $\mathrm{P}(B)=p_{0}$ is $\mathrm{PC}$ on $B, \mathrm{P}(B \mid B-x)$ is the $\mathrm{PC}$ on $B$ in the condition of skipping $x$, $\mathrm{P}(B-x)=1-g(\mathrm{x})$ is $\mathrm{PC}$ on " $B$ without $x$ " and is the same with pne $e_{x} \mathrm{P}(B \cup B-x)$ is the $\mathrm{PC}$ on $B$ both with $x$ and without $x$, that is, $\mathrm{P}(B)$. Otherwise, $x$ is put back to $B$ if is not skipped. Then, a new block is randomly selected from the remaining blocks iteratively until the updated confidence is equal to or is very close to 1 . Finally, the blocks in $B$ are the candidate set for scanning.

$\mathrm{H}$-selection removes the blocks whose PEs are lower than the confidence (blocks with small PE), instead of only selecting the blocks whose PEs are higher than the confidence (blocks with larger PEs). There are two reasons for this strategy. First, the design of $g(x)$ ensures the rule like "more blocks with low PEs and few blocks with high PEs," therefore, a lot of blocks are skipped in the scan operation even if the confidence is relatively large. Second, because blocks with low PEs are far more than those with high PEs, skipping low-PE-blocks gains better randomness than selecting high-PE-blocks. For example, when a query is executed repeatedly, the $\mathrm{H}$-selection algorithm with a skipping mechanism may skip different low-PE-blocks each time, which the algorithm with a selecting mechanism may select the same high-PE-blocks.

There are two exceptions that $\mathrm{H}$-selection cannot guarantee that the PC is less than the confidence. First, the confidence is set to be a very small value. Second, all blocks have very small PEs. In these two situations, the condition " $p_{0} \approx 1$ " in Algorithm 2 may not be satisfied even if all blocks are skipped. For the first situation, PoBery has a forced stop mechanism to prevent too many blocks from being skipped if the confidence is set to be zero or an extremely small value. The second situation never happens because, as discussed in the previous two sections, the function $f(x)$ and $g(x)$ are well designed to be full of the possibility space, and there are enough records in a cell to be placed ( $\omega$ is not a very small value).

\section{ARCHITECTURE}

In this section, the software architecture of PoBery is explained. PoBery is implemented in Java 1.8. As shown in Figure 9, it requires two host systems: Drill (v 1.13) as a query engine and HDFS 


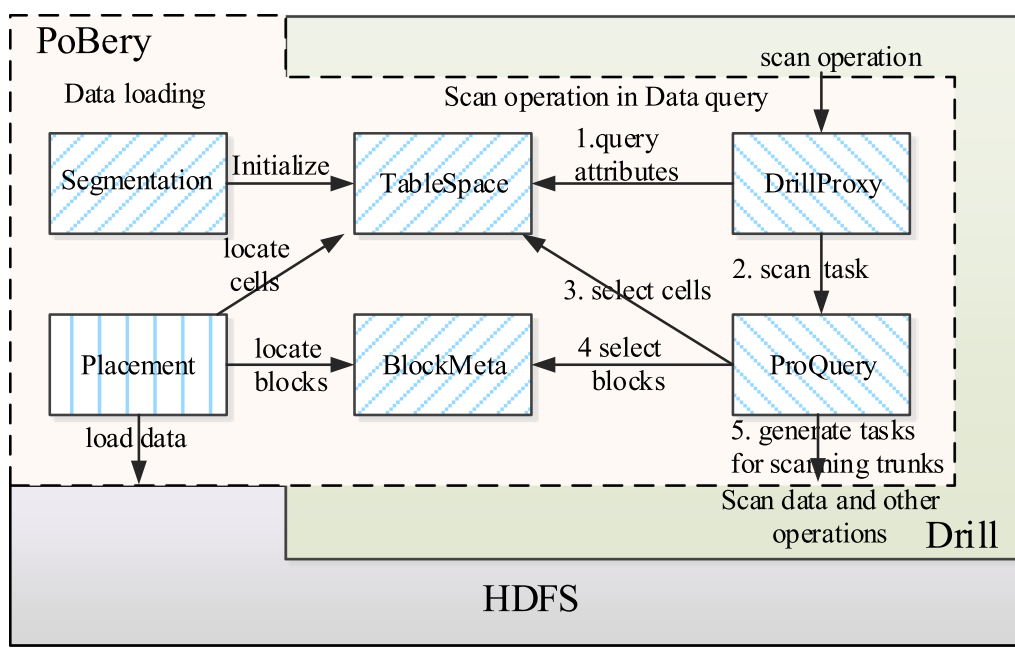

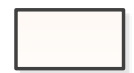

PoBery

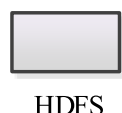

HDFS

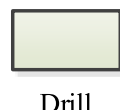

Drill

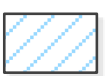

Modeling

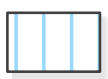

Loading

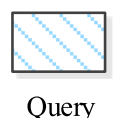

Query

Fig. 9. Software architecture of PoBery.

(Hadoop Distributed File System) (v 2.4) [12] as a distributed file system. It inherits most of the features of the host systems. Different modules collaborate to implement the tablespace, probabilic data placement, and query. These modules include DrillProxy, TableSpace, BlockMeta, Placement, Segmentation, and ProQuery.

- TableSpace: TableSpace is a tree-structural data model implemented with an in-memory data structure on the master node.

- Segmentation: Based on prior knowledge, segmentation defines query attributes and partitions them into near-equal-frequency intervals according to their value range.

- DrillProxy: When DrillProxy receives a query from Drill, it first validates the query based on TableSpace, then submits the query to ProQuery.

- BlockMeta: BlockMeta calculates and stores the PPs and PEs of all blocks. It is the metadata of files in storage.

- ProQuery: ProQuery is a core component of PoBery that implements the strategies and algorithms in Section 5.

- Placement: Placement is the other core component of PoBery. Based on BlockMeta, it places data into chunks of blocks, as described in Section 4.

- Query Engine (Drill). PoBery can support other "SQL on Hadoop" query engines. In this article, we employ the Drill-based query engine to facilitate discussion.

- Storage. Key-values data is stored on a Hadoop HDFS. PoBery blocks are modeled as HDFS files, and PoBery trunks are modeled as HDFS blocks.

\section{EXPERIMENTS}

\subsection{Purposes}

The experimental purposes have three parts: First, studying the effectiveness and efficiency of the data model; second, validating the design purposes of PoBery; and third, studying the performance 
of PoBery and comparing it with other "SQL on Hadoop" systems. We start with the conclusions of each experiment, as follows.

Section 7.3. The creation and traverses of a tablespace are efficient. The performance is related to the number of query attributes (levels) but unrelated to the data distribution. The cost of the tablespace is negligible.

Section 7.4. The additional cost of data placement is slight and acceptable; the data amounts as results of data placement on each node are balanced.

Section 7.5. PoBery meets the promise that the percentage of complete queries is larger than the given confidence for various cases.

Section 7.6. PoBery meets the promise that the query speedup is obvious when PC slightly decreases, while speedup tends to stabilize when PC greatly decreases.

Section 7.7. Compared with Drill and other "SQL on Hadoop" systems, the query performance of PoBery doubles at the cost of a slight PC loss, while the query performance remains unchanged if the confidence is set to be 1 , under the various query conditions and data amounts.

\subsection{Setup}

Experiment environment. We run experiments on a cluster with 12 physical machines. Each machine has the same specifications: Intel Core i7, 1 TB hard disk, 8 GB memory, and CentOS 7. The gigabit Ethernet is connected using a Dell PowerConnect 5548.

Experimental data. We adopt table UserVisits in HiBench [13]. The schema of UserVisits are sourceIP (varchar), destURL(varchar), visitDate (date), adRevenue(float), userAgent(varchar), countryCode(char), languageCode(char), searchWord(varchar), duration(int). The data generator is from HiBench and the data amount is a config in hibench.join.bigdata.uservisits. There are maximum 1,000 records in a trunk. We set the number of blocks to be $2,000\left(B_{1}\right), 4,000\left(B_{2}\right), 6,000\left(B_{3}\right), 8,000$ $\left(B_{4}\right)$ and $10,000\left(B_{5}\right)$. The scale of datasets are $20\left(D_{1}\right), 40\left(D_{2}\right), 60\left(D_{3}\right), 80\left(D_{4}\right)$, and $100\left(D_{5}\right)$ million records; the data size is about $200 \mathrm{MB}$ per million records. This way, all blocks contain an average of 10,000 (10 trunks) to 50,000 (50 trunks) records with balanced data distribution among the blocks. The sorting algorithm on datasets is Terasort [14].

Tablespace. In Section 7.3, we plan the more diversiform tablespaces with UserVisits. For the other experiments, the tablespace contains three levels. They are visitDate, adRevenue, and sourceIP from top to bottom. The number of cells is approximately equal to the number of blocks, from $B_{1}$ to $B_{5}$.

Query cases. The query case contains three query attributes as the conditions: visitDate, adRevenue, and sourceIP. For highlighting our proposition in scan operation, we design the query cases without aggregation, join, sort, and other operations. Otherwise, the performance difference is hidden by these mechanisms. In all experiments, there are three strategies of generating query conditions, as follows.

For Section 7.3, we plan to enumerate all kinds of the traverse on the tablespace. To this end, we design two strategies. In the strategy of "containing," the query condition is a record that is randomly selected from the dataset. In the strategy of "joint," each query attribute has a $60 \%$ percent of chance to be selected, and its condition randomly covers $10 \%$ percent of the range on the attribute.

For Sections 7.5 and 7.6, we need to summarize the results easily. To this end, the query conditions are ranges defined by a random segment on each level in the tablespace. The confidences are from 0.1 to 0.9 , and denoted as $C_{1}$ to $C_{9}$.

For Section 7.7, we compare the performance of PoBery and Drill in various query scales. To this end, we control the expected percent of segments in each level of a tablespace for a query. The 
Table 2. Symbols Used in Experiment Illustration

\begin{tabular}{ll}
\hline Symbol & \multicolumn{1}{c}{ Description } \\
\hline$B_{i} D_{j} C_{k}$ & $\begin{array}{l}\text { A test case that refers to the query on } B_{j} \text { number of blocks, } D_{j} \text { dataset, and } \\
\text { specified confidence } C_{k} . i \text { and } j \text { are in }\{1,2, \ldots, 5\} \text { and } k \text { is in }\{1,2, \ldots, 9\} .\end{array}$ \\
$\operatorname{opc}\left(B_{i} D_{j} C_{k}\right)$ & $\begin{array}{l}\text { The observed PC of the case } B_{i} D_{j} C_{k} \text {. PoBery expects that it is larger but close to } \\
k / 10 .\end{array}$ \\
& $\begin{array}{l}\text { The scan efficiency (SE) of } B_{i} D_{j} C_{k} \text {, which is the ratio between the number of } \\
\text { blocks that accurately contain the queried records, and the number of blocks } \\
\text { that are selected to be scanned. }\end{array}$ \\
\end{tabular}

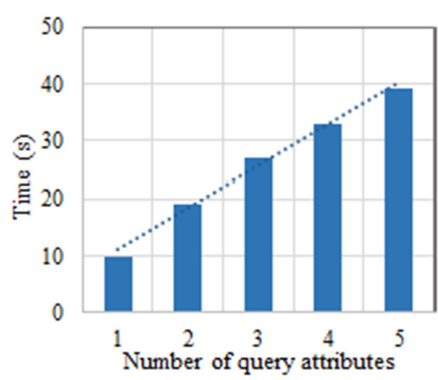

(a)

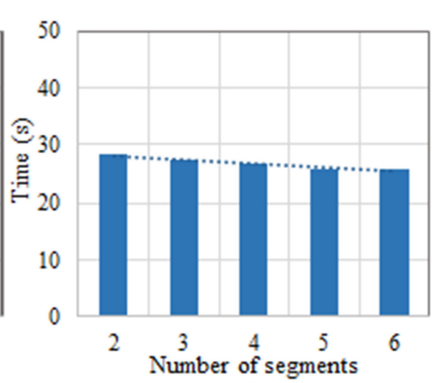

(b)

Fig. 10. The effect of parameters on the creation time of Tablespace.

ratio between matched segments and all segments in a level, denoted as $r$, varies from 0.1 to 0.7 . Therefore, the query scale, as the ratio between the result-set size and the dataset size, is $r^{3}$, that is, query scales are $0.001(r=0.1), 0.008(r=0.2), 0.027(r=0.3), 0.064(r=0.4), 0.125(r=0.5), 0.216$ $(r=0.6)$, and $0.343(r=0.7)$.

Symbols. Table 2 summarizes the symbols used in the discussion and analysis of the experiments.

\subsection{Model Analysis}

There are two models with many parameters in PoBery, the data model and the probability model. The data model's parameters are the number of query attributes $z$, the number of segments $\kappa$, and the distribution of data. The probability model's parameters are the number of records $\omega$ per slot (also placing time per slot), a selected positive number $\lambda$, the number of blocks $n$, and $\sigma$ and $\mu$ as two parameters of a normal distribution function. The parameters of the probability model are manually specified according to the expected feature of $f(x)$ and $g(x)$. They are analyzed in Sections 7.5 and 7.6.

In the data model, we study the creation time and traverse time of a tablespace. Both are related to the structure of the tablespace, which is dominated by $\mathrm{z}$ and $\kappa$. In the tablespace creation, the key step is equal-frequency binning. It sorts the data first and then partitions them by quantity [9]. Therefore, data distributions of query attributes do not affect binning performance. Sometimes, the extremely skewed data distribution may result in an approximate equal-frequency segment. However, PoBery is tolerant of such approximation. To study the impacts of $z$ and $\kappa$, we plan two experiments with the $D_{5}$ dataset (100 million). The first creates tablespaces whose $z$ is 1 to 5 and $\kappa=4$. The results are shown in Figure 10(a). The second creates tablespaces whose $\kappa$ is 1 to 5 and $z=4$. The results are shown in Figure 10(b). First, the creation time of each case is 


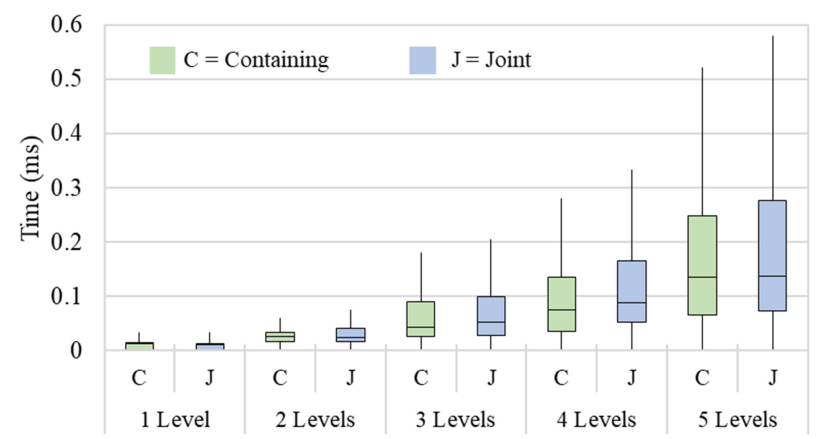

Fig. 11. Comparison of cell selection time in different query modes.

less than 1 minute. It is acceptable because the tablespace creation runs only once. In addition, the creation time would reduce if we sample records data rather than sort all of them. Second, it increases linearly with the number of query attributes-namely, the levels of tablespace-and slightly decreases with the increase of segment amount. On the one hand, if there is one query attribute, the creation time is mainly a single time of Terasort [14] on the attribute; then, adding one level brings an additional Terasort on the new attribute. Therefore, Figure 10(a) shows a linear growth trend. On the other hand, the parallelism of Terasort [15] on the none-first-level attribute is better if its parent level has more segments. Therefore, Figure 10(b) shows a slight decrease trend.

PoBery traverses a tablespace in two operations. The first is in data placement. When a record is placed, PoBery traverses on a tablespace for the cell it belongs to. It is a "containing traverse" because the condition is one record, that is, which cell contains the record. The second is in scanning. Given a query condition, PoBery traverses on tablespace for cells it covers. It is a "joint traverse" because the condition is a range, that is, which cells have an intersection with the given range. In the experiment, we study the performance of these two traverses on tablespaces mentioned in Figure 10(a). The test cases follow the strategy of "containing" and "joint" mentioned in Section 7.2. We generate 1,000 cases and the summarization of traverse time is shown in Figure 11. First, the traverse time is about 0.5 millisecond, which is negligible compared with the second-level data query (see also Section 7.6). Second, it increases with the number of levels because the tablespace structure becomes more complex when it has more levels. The time of containing traverse is less than that of joint traverse because the former filters out more segments in top levels than the latter.

\subsection{Data Placement}

Data placement, which determines the target block for a record, is a critical process before PoBery loads data to storage. Figure 12 shows the average time taken by the DPA (Data Placement Algorithm) to process various block numbers and data volumes. Overall, the DPA takes no more than 5 seconds to process 100 million records. On the one hand, DPA does not incur significant costs when loading data, compared with the cost of I/O operations. For example, if data loading is performed with two threads, one of which places data and the other loads data, the former thread never blocks the latter one. Therefore, data loading performance is dominated by I/O performance. The cost of the DPA is negligible. On the other hand, data loading is performed only once and all the queries benefit from the probabilistic data placement. Thus, the loading cost is offset by query speedup. Overall, the extra cost incurred the by DPA in data loading is negligible.

As shown in Figure 12, there is a linear and positive correlation between execution time and data volume. Given the same data volume, the execution time of DPA slightly increases with the number of blocks because PoBery takes more effort to map probabilities to block indexes. In our 


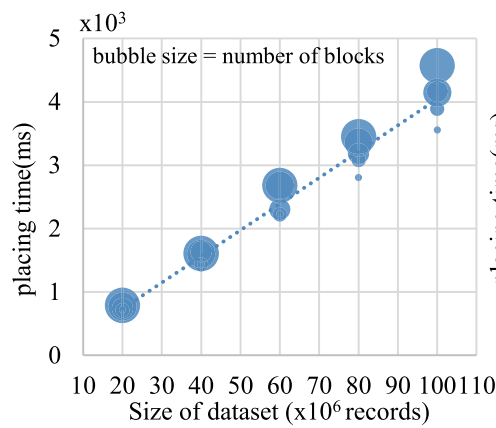

(a) DPA time of different dataset volumes

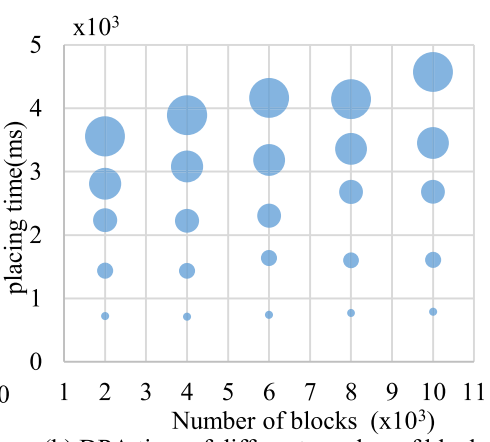

(b) DPA time of different number of blocks

Fig. 12. Comparison of DPA time of different cases.

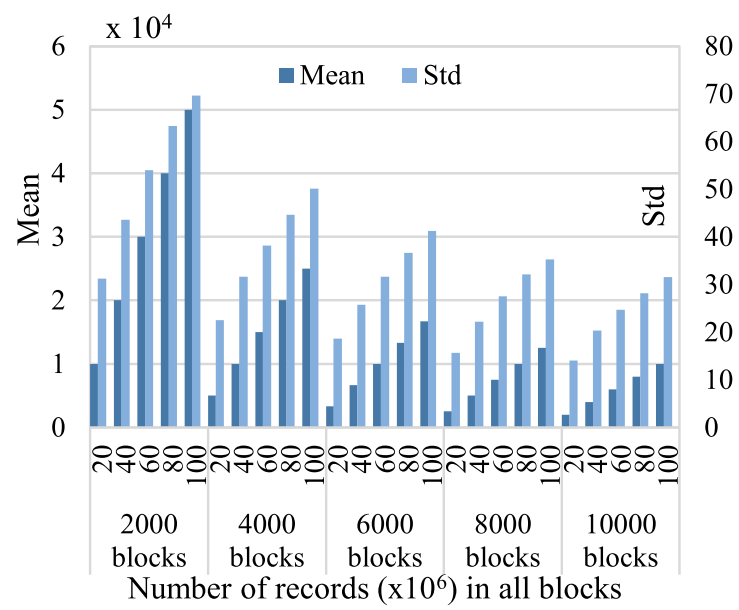

Fig. 13. The data distribution among blocks (mean and standard deviation).

Java implementation, we do not adopt org.apache.commons.math3.distribution.NormalDistribution to calculate probability or cumulative probability due to its low performance. Instead, a lookup table is initialized and $f^{1}(x)$ is implemented as a binary search on the table. When there are more blocks, the table is larger and the binary search is slightly more expensive.

To validate that the data distributed in each block is balanced, we calculate the mean and standard deviation of the data volumes in blocks under the 25 combinations of $B_{i} D_{j}$. Figure 13 shows the results. The mean values, denoted by dark bars and primary axis, equal to the data volume divided by the number of blocks. The standard deviations, denoted by light gray bars and secondary axis, are positively correlated to the mean values but never exceed 80 . This indicates that the data volume distributed in the blocks is balanced.

\subsection{Observed PC and Completeness}

In PoBery, we expect that the observed PC, opc $\left(B_{i} D_{j} C_{k}\right)$, is large and close to $k / 10$ ( $k$ is the subscript of $C$ ). The observed PC of specific confidence is summarized using 1,000 queries. Figure 14 shows the $50^{*} 5$ observed PCs grouped by $D_{j}$ first and $B_{i}$ later. From Figure 14, the following observations are drawn: 

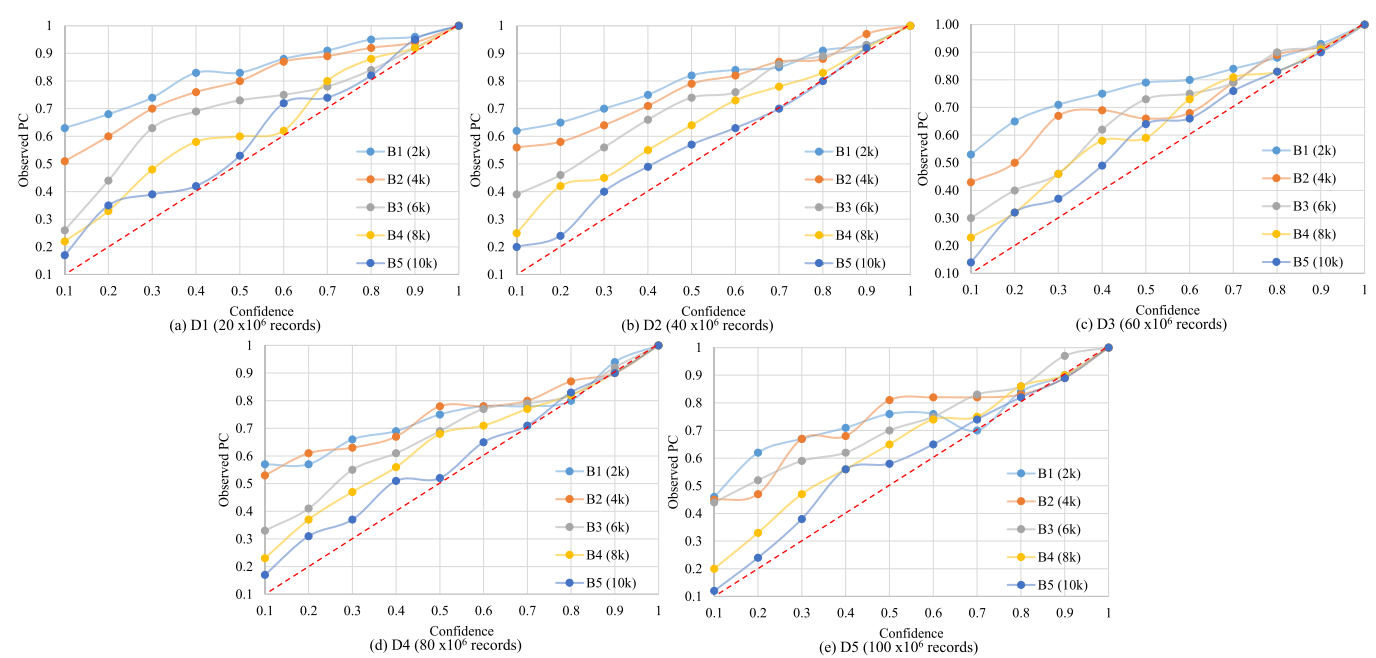

Fig. 14. Relationship between observed PCs and confidences in various cases.

(1) In all cases, the observed PCs are no smaller than the specified confidences because each curve, which represents the relationship between the observed PC $(y)$ and confidences $(x)$, is not below the reference line $(y=x)$.

(2) In most cases, the observed PC increases with confidence because the completeness increases monotonically. However, there are some exceptions, such as $B_{2} D_{3}$ in Figure $14(\mathrm{c})$, and $B_{5} D_{5}$ in Figure 14(e). Because the H-selection algorithm selects blocks randomly, sometimes the floating of PE causes the situation that the observed PC for confidence $x_{1}$ is larger than that for confidence $x_{2}$ despite that $x_{1}$ is slightly lower than $x_{2}$.

(3) The observed PCs do not exactly obey the confidences. In all cases, the curves end at the range $(1,1)$, but start from the range $(0.1,0.6)$. In other words, when the confidence increases from 0.1 to 1 , there are some cases in which the observed PC quickly increases from 0.1 to 1 , while in other cases the observed PC increases slowly from 0.5 to 1 . Whether the observed PC increases quickly or slowly is dependent on the number of blocks but not the data volume. If there are more blocks, their PEs are closer. This indicates that the granularity of PE distributed on these blocks is finer. Accordingly, H-selection has more chances to select blocks with different PEs. In contrast, PEs concentrates on a few distinct values if there are a small number of blocks. Thus, the observed PCs are close no matter what the specified confidences are.

(4) The best cases that satisfy the design purposes of PoBery are $B_{5} D_{j}$, because their curves are close to the reference line $(y=x)$ most of the time. We believe that $f(x)$ suits $B_{5}$ well, when $\sigma=$ 0.3989 and $\mu=2$. Actually, we can also define $f(x)$ with different $\mu$ to adapt $B_{1}$ to $B_{4}$ better than the current $\mu$ value. However, $f(x)$ with a large $\mu$ raises the problem of "too many 0 s in $g(x)$." In addition, $f(x)$ with a small $\mu$ raises the problem of "values of $g(x)$ are similar," as demonstrated in Figure 15. In practice, the number of blocks is fixed so that a carefully designed $f(x)$ is beneficial.

As the observed PC meets the promise, we further study the observed query completeness. For a query case executed 1,000 times, there are incomplete results if $\mathrm{PC} \neq 1$. However, the incomplete one loses a little completeness. The minimum observed completeness of all cases for 1,000 executions is 0.9734 , namely, for millions of data queried, at most $3 \%$ of them are missed. Figure 16 shows the minimum observed completeness of 1,000 executions for each query case, sorted increasingly. A total of $66 \%$ are larger than 0.995 ; therefore, the accuracy of incomplete queries is much "closer" to complete queries. The next section shows that many blocks are skipped when PC $\neq 1$. However, 


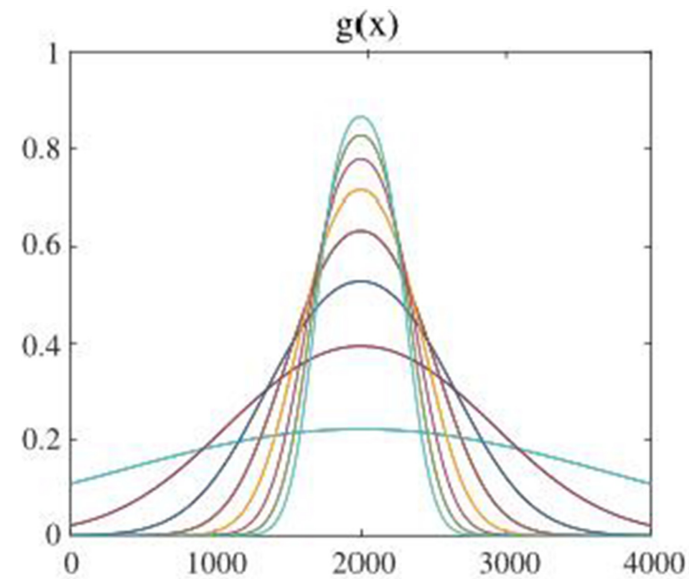

Fig. 15. Image of $g(x)$, curves ordered by their peaks are $\mu=0.5,1,1.5,2,2.5,3,3.5$, 4, where $\omega=1,000, \mathrm{n}=$ 4,000 , and $\sigma=0.3989$.

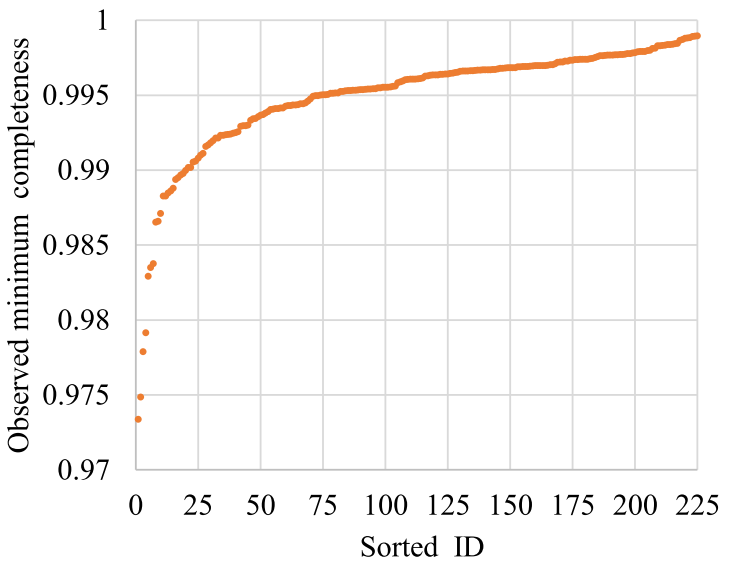

Fig. 16. The observed minimum completeness of 225 query cases sorted increasingly.

the skipped blocks likely have small PEs for the queried records; thus, the observed completeness is closer to 1 even when they are skipped.

\subsection{Query Efficiency}

PoBery trades off the possibility of query completeness for query performance. In this experiment, we define the scan efficiency (SE) to indicate the performance speedup when the confidence decreases. SE is the proportion of matched blocks among the scanned blocks. It is the ratio between the number of blocks that accurately contain the queried records and the number of blocks that are selected to be scanned. The theoretical maximum $\mathrm{SE}=1$, namely, all selected blocks contain the queried records. However, it can never be reached in practice. We expect that $\operatorname{se}\left(B_{i} D_{j} C_{x}\right)>$ $s e\left(B_{i} D_{j} C_{y}\right)$ if $x<y$, namely, the loss of PC trades the query performance. There are a total of 250 combinations of test cases; we summarize the five-statistical numbers (min, Q1, median, Q2, max) for 1,000 queries for each case. Figure 17 shows the results. From Figure 17, the following observations are made. 

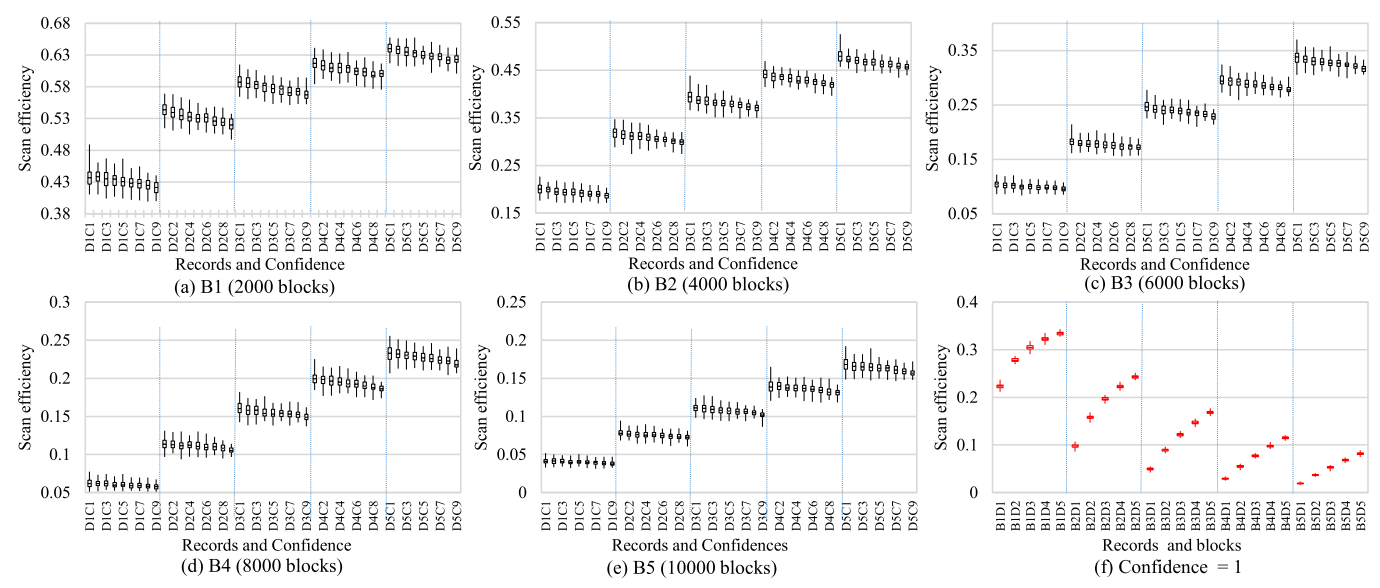

Fig. 17. SE of possibly-complete query cases (a) to (e), comparing with SE of complete query cases (f), i.e., confidence $=1$.

(1) In all the cases, the SEs of the possibly-complete queries are definitely higher than complete queries (confidence $=1$ ), as shown by Figure 17(a), (b), (c), (d), (e) to Figure 17(f). While for each case with the same $B_{i} D_{j}$, SE decreases with the confidence because fewer blocks are skipped. However, it does not decrease proportionally because the PEs of almost half of the blocks are neither 0 nor 1 (see Figure $15 u=4$ ). Thus, $\mathrm{H}$-selection is performed on the remaining blocks. The SEs of different confidences are similar because there are too many $1 \mathrm{~s}$ and $0 \mathrm{~s}$ of PEs.

(2) Given the same number of blocks, SE increases with the data volume, i.e., $\operatorname{se}\left(B_{i} D_{x}\right)>\operatorname{se}\left(B_{i} D_{y}\right)$ if $x>y$. Given the same data volume, SE increases when the number of blocks decreases, i.e., $s e\left(B_{x} D_{j}\right)>s e\left(B_{y} D_{j}\right)$ if $x<y$. These regularities are also satisfied when confidence $=1$, as shown in Figure 17(f). In this experiment, the SE fluctuation is dominated by the queries themselves but not the strategies of PoBery. Each block contains more records when there are more data but the number of blocks is unchanged, as well as there are fewer blocks but the data volume is unchanged, thus more selected blocks contain the queried records.

\subsection{System Comparison}

We select Apache Drill as the query engine of PoBery because it is more up-to-date than MapReduce. We set PoBery with different confidences, that is, 1, 0.8, 0.5, and 0.2, denoted as PoBery1, PoBery0.8, PoBery0.5, PoBery0.2, respectively. We compare its query performance with Drill, Impala, and Hive on Tez. They are all "SQL on Hadoop" systems [16]. The former two are based on MPP and Apache Parquet [17] while the latter is based on the DAG framework [18] and ORC [19]. Because Impala and Hive have different query optimization approaches, we treat them as two baselines in the comparison. Generally, Impala is faster than Drill for its high I/O performance [20], and Hive-Tez is faster than Hive-MR. In particular, inline indexes in ORC files make Hive even more efficient. In our experiment, Hive sorts one of the queried attributes, adRevenue, to utilize the inline index of OCR files.

PoBery employes the possible incomplete query and the other three systems employ the complete query. However, the performance comparison is fair for the following three reasons:

(1) As shown in Figure 16, the minimum observed completeness of 1,000 executions for each query case is around 0.995 . It is very close to the complete query, that is, for the query in Drill, Impala, and Hive, missing $0.5 \%$ matched records does not greatly improve the performance, especially considering the offset of query parallelism. 


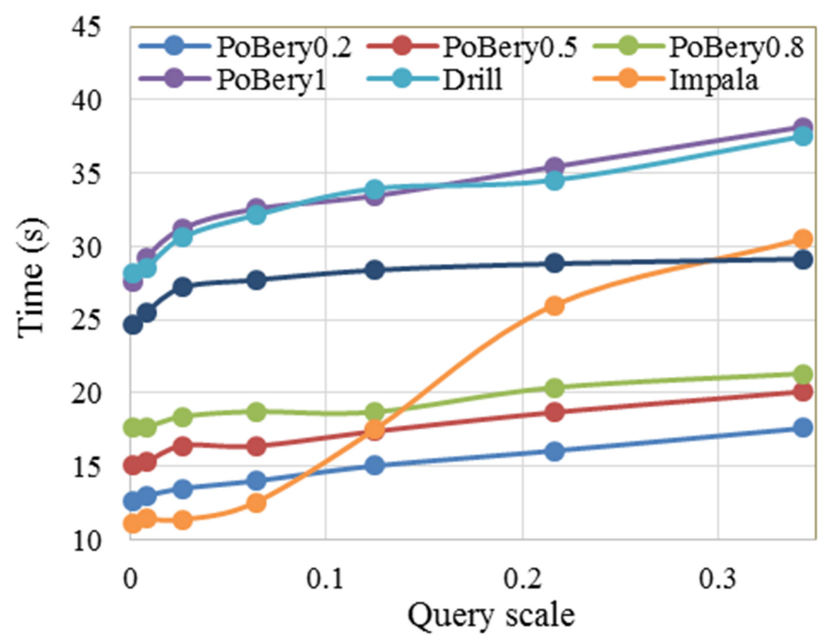

Fig. 18. Query time on different query scales.

(2) In PoBery, we repeat each query case 1,000 times, and some are incomplete. The query performance of the case is the average execution time; therefore, the benefit of incomplete ones is offset.

(3) Both four system employs a full-scan but not a partition or sharding techniques. PoBery does not partition any data, and records in a cell may be placed in any blocks, the same as Drill, Impala, and Hive. During the scan, PoBery adopts H-selection to skip the block with lower PE, while Hive adopts the inline indexes of OCR files (Predicate Pushdown), and Impala adopts a dictionary page in a parquet block to try to identify whether all of the blocks can be skipped all at once.

Figure 18 shows the query times taken different approaches on different query scales, and the following observations are drawn:

(1) The experiment is conducted on the $20 \mathrm{~GB}$ dataset. The query scales are $0.001(r=1), 0.008$ $(r=2), 0.027(r=0.3), 0.064(r=0.4), 0.125(r=0.5), 0.216(r=0.6)$, and $0.343(r=0.7)$. The results show that, for all scales, the performance of PoBery1 is the same as Drill and close to Hive, but Prober0.x is faster than Drill and Hive; for scales $>0.15$, Prober0. $x$ is faster than Impala.

(2) The curves are almost stable except for that of Impala. It shows that the number of scanned blocks is independent of the size of query ranges, different from the data partition mechanism. Given more records in the query results, the output operation takes more time. This explains why the curves are slightly upward. Impala utilizes memory to store intermediate results and offers a spill-to-disk operation to avoid exceeding the memory limit. For the larger result sets, frequent spill-to-disk operations decrease performance.

Figure 19 shows the performance and scalability of PoBery to Drill, Impala, and Hive. Query performance and scalability of PoBery1 and Drill are the same because PoBery is based on Apache Drill, and it scans all blocks when confidence $=1$. PoBery0. $\mathrm{x}$ is faster than the other three systems. Further, the following observations are drawn.

(1) For all datasets, the performance of PoBery0.8, PoBery0.5, and PoBery0.2 increase gradually, while the performance of PoBery1 and Drill are almost the same. PoBery meets the design goals and optimization goals.

(2) PoBery has sublinear scalability to the dataset volume. This is inherited from Drill. Impala shows very stable performance on the $D_{1} \sim D_{3}$ datasets but turns worse on the $D_{4}$ and $D_{5}$ datasets because of the memory limitation. PoBery does not rely on pre-computation or materialized techniques. Its in-memory data structure, tablespaces, contains the metadata of levels and segments 


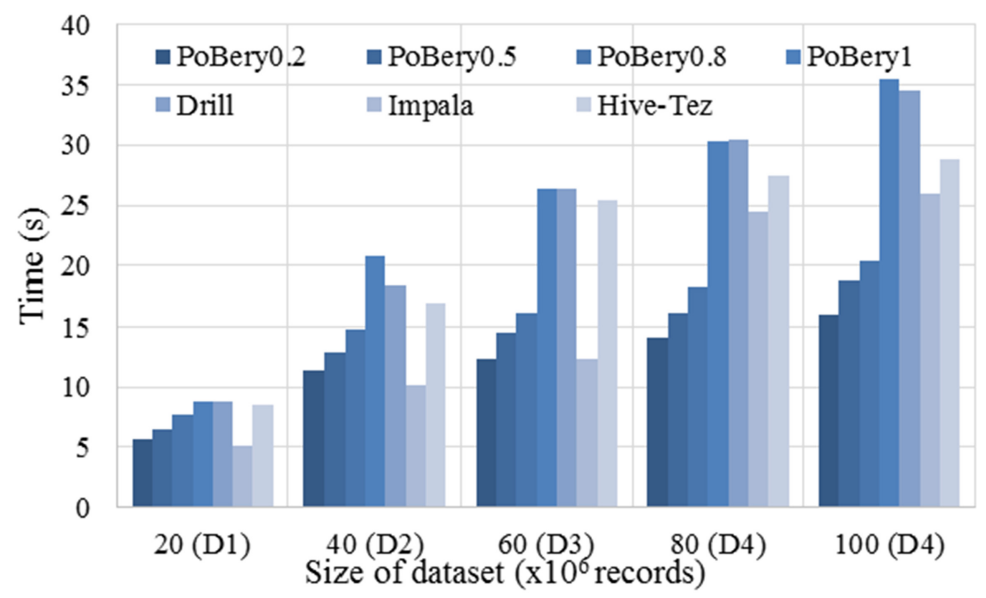

Fig. 19. Query time on five datasets. The bars represent PoBery0.2, PoBery0.5, PoBery0.8, PoBery1, Drill, Impala and Hive-Tez from the left to the right.

only. The mapping relationship between cells and blocks is calculated but not searched. As a result, the memory is saved. Impala is slightly faster than PoBery0.x on the $D_{1} \sim D_{3}$ dataset, and PoBery0.x catches up with Impala on the $D_{4}$ and $D_{5}$ datasets.

\section{RELATED WORKS}

The problem of effectively and efficiently querying data in a probabilistic way has recently gained a great deal of attention from the big data research community. The main reasons are twofold. First, there are increasingly broad and various applications where uncertain or incomplete data need to be processed. Second, there is an urgent need for trading off between imprecise or incomplete query results and query performance in these big data applications. To the best of our knowledge, PoBery is the only solution for processing possibly-complete queries where the users can set to what extent their queries may be complete to pursue fast responses by trading off PC. Similarly, BlinkDB [21] trades off result accuracy for faster response time by running precise queries on data samples. Merlin [22] trades off the accuracy of probability estimation on approximate queries for faster response time. The concept of "incomplete queries" against the queries over "incomplete data" studied in [23] is slightly similar to the concept of possibly-complete queries proposed in this article. BlinkDB [21] and Merlin [22] support "approximate queries" against the query over "approximate data." Thus, we categorize the related work into four groups: (1) query relaxation that is close to incomplete query; (2) query over incomplete data; (3) approximate query; and (4) query over uncertain (approximate) data.

(1) Query Relaxation. Interactive Data Exploration (IDE) is a key ingredient of a diverse set of discovery-oriented applications, for example, scientific computing and evidence-based medicine applications [24]. In these applications, data discovery is a highly ad hoc, interactive process in which users execute numerous exploration queries using varying predicates aiming to balance the trade-off between collecting all relevant information and reducing the size of returned data [25]. Query relaxation enables applications to execute the most restrictive version of a query first and progressively relax the query until the required number of hits is obtained [26]. It pursues query completeness with flexible query conditions. However, the cost is low performance. Representative work in this category includes interactive query relaxation [27] and approximate query relaxation [28]. 
An alternative approach for improving the response time of exploratory queries is to present approximate results, such as online processing techniques [29] and interactive, intuitive techniques for analyzing massive datasets [30]. These techniques offer approximate answers, and their goal is to allow users to obtain a quick sense of whether their queries reveal anything interesting about the data. PoBery works in a way similar to IDE. For example, when a user submits a query with a very small confidence and immediately obtains the results, the user takes a glance at the results and resubmits the same query with a larger confidence if the previous results contain interesting but inadequate results. Meanwhile, PoBery supports both range queries and aggregated queries as described in [31]. PoBery also offers the capabilities of "discover data" and "analyze data" by further processing the result set with "possible completeness."

PoBery is different from query relaxation because finding query results with larger confidence is more comprehensive than finding results with smaller confidence. The crucial assumptions underlying query relaxation approaches are that the users desire complete queries and they can explicitly state whether their queries are complete. PoBery, however, allows users to not state the complete results and they desire guarantees about the possibility and latency beforehand.

(2) Query Over Incomplete Data. Incomplete databases are presented in many research domains. The relevant work focuses on dealing with incomplete data with incomplete data models, incomplete data indexes, incomplete skyline and similarity search, and so on. [32]. There are three main methods for processing incomplete data: the simple deletion method, the missing value imputation approach, and the special treatment method. The first two try to complete incomplete datasets. In contrast, special treatment methods tackle incomplete datasets directly, such as the new ISQL [33], the new dominance relationship definition on incomplete data [34], the distance function for incomplete objects [35], and the probability estimation on incomplete data [36]. In [36], a probability model is proposed to estimate the similarity between a query and the data objects with missing dimensions. PoBery does not process the incomplete data but rather focuses on possibly-complete queries. PoBery adopts a probability model to estimate the existence of the queried data objects and storage locations. Thus, similar to the method proposed in [36], PoBery guarantees the possibility of query completeness.

(3) Approximate Query. An approximate query is characterized by its core algorithm, its error model and accuracy guarantee, the amount of work saved at runtime, and the amount of additional resources it requires in pre-computation. In recent studies of big data queries, approximate queries are used to provide an optimization method for traditional query methods in various situations, such as A/B testing, hypothesis testing, exploratory analytics [37], feature selection [38], and big data visualization [39, 40]. BlinkDB [21] is an approximate query processing framework for running interactive queries on large volumes of data. Soon after the commercialization of BlinkDB by Databricks [41], several other Big Data query engines also started to add support for approximation features in their query engines, such as SnappyData [42] and Facebook's Presto [43].

Approximate query results are as useful as their accuracy guarantee. Quantifying or estimating the extent of errors in approximate results is performed not only with variance or bias but also with a probability of existence. Bias and variance are used to assess the approximation quality of a numerical estimate. However, in the case in which a query result is not a single number but rather a relation, the probability of existence of an output tuple is the probability with which it also appears in the output of the exact query. The probability of existence is well studied in the context of probabilistic databases [44] and can be easily computed using bootstrapping [45]. PoBery also defines the probability of existence (see Definition 6) to evaluate the probability of whether a block contains the data and to determine whether a block is included in the scan scope. 
Sampling is another approach for approximating queries. The query results may not be accurate but still representative. Sampling has a long history in databases-over two decades [46, 47]. Users can perform sampling over query results, base tables, or the entire database [48]. Among sampling methods, probability sampling ensures that every unit in the population has a chance (greater than zero) of being selected and included in the sample. This probability can be accurately determined. PoBery adopts a similar technique in data placement. It selects the target block to place data according to a certain probability. However, sampling-based approximation queries are orthogonal to our problem. Due to sampling, the query results are certainly incomplete, and the probability measures the extent of such incompleteness. PoBery's query results are possibly incomplete, and the probability measures such possibility.

(4) Query Over Uncertain Data. Uncertain data are those that deviate from the original values because of the noise in the data. A probabilistic database is an uncertain database in which the data have associated probabilities [49]. There are two kinds of uncertainty: "membership uncertainty" (tuple-level) [44, 50] and "value uncertainty" (attribute level) [51, 52]. The former treats tuples as uncertain events capturing the belief that they belong to the database. The latter usually represents attributes with a discrete or continuous probability distribution depending on the data domain. PoBery is inspired by the concept of membership uncertainty and then associates the "placementship uncertainty" with data placement, that is, the data placing to the storage location is uncertain.

Query processing on uncertain data includes range queries, threshold queries, skyline queries, top-k queries, nearest-neighbor queries, aggregate queries, and join queries. The first two inspire PoBery. A probabilistic range query tries to retrieve the results with the form of $(o, p)$, where $p$ is the probability of object $o$ satisfying the query conditions [53]. A threshold query retrieves the objects qualifying a set of predicates with certain threshold guarantees. A range query processing in PoBery includes two steps. Frist, PoBery finds the logic container of the query target denoted as cell and then calculates $(b, p)$, where $b$ is a storage location called block and $p$ is the probability of the cell stored on the block. This step is an analogy to the probabilistic range query. Second, a heuristic block-selection algorithm (see Section 5) is performed on blocks with a given confidence. This step is analogous to the threshold probability query.

\section{CONCLUSION}

This article presents the design, implementation, and evaluation of PoBery, a possibly-complete query supported by probabilistic data placement and scanning. PoBery is built on a Hadoop HDFS, and Drill as a query engine. PoBery employs the models of tablespaces, segments, cells, blocks, and trunks, as well as the probability of query completeness (PC), the probability of existence (PE), and the probability of placement (PP). Based on these models, the data placement algorithm ensures both the uneven probability distribution and balance data distribution, and parallelism of data scanning. The cell and block selection algorithms in the scan process skip the blocks with small $\mathrm{PE}$ according to the query conditions.

PoBery has some advantages: (1) The data model and data placement algorithm are efficient. The additional cost is negligible. The possibility of query completeness and query efficiency meet design purposes. (2) PoBery guarantees that the observed PC is not less than the expected PC. PoBery randomly skips the data blocks with small PEs and greatly improves the efficiency of data scanning. (3) Among the incomplete query results, the query completeness is also larger, namely, there is only a few losses of query completeness; (4) For the Drill-based implementation, PoBery performs as fast as Drill with complete queries, while performing, on average, $1.8 \times, 1.3 \times$, and $1.6 \times$ faster than Drill, Impala, and Hive on Tez with possibly-complete queries, respectively.

In future studies, PoBery will be further optimized in several ways. We will further investigate the $f(x)$ employed by PoBery's DPA because its optimization performance is dominated by a 
well-designed $f(x)$. Currently, PoBery does not leverage the sophisticated data structures for the storage data in chunks but rather remains the file format of the host system. It would facilitate the data scanning if a PE index is stored in each data file. We will also integrate PoBery with other fashionable query engines, such as Spark-SQL and Hive-HQL.

\section{ACKNOWLEDGMENTS}

The authors thank Wu Jingbo and Hou Yaowei, two graduate students of Software College, Northeastern University, for the initial work on PoBery and the revision work on the draft, respectively.

\section{REFERENCES}

[1] John Klein, Ian Gorton, Neil Ernst, Patrick Donohoe, Kim Pham, and Chrisjan Matser. 2015. Performance evaluation of NoSQL databases: A case study. In Proceedings of the 1st Workshop on Performance Analysis of Big Data Systems, Austin, Texas, USA. ACM, 5-10.

[2] Badrish Chandramouli, Guna Prasaad, Donald Kossmann, Justin Levandoski, James Hunter, and Mike Barnett. 2018. FASTER: A concurrent key-value store with in-place updates. In Proceedings of SIGMOD'18. New York, NY, 275-290.

[3] Song Jingkuan, Yi Yang, Xuelong Li, Zi Huang, and Yang Yang. 2014. Robust hashing with local models for approximate similarity search. IEEE Transactions on Cybernetics 44, 7 (2014), 1225-1236.

[4] Brian Babcock, Surajit Chaudhuri, and Gautam Das. 2003. Dynamic sample selection for approximate query processing. In Proceedings of the 2003 ACM SIGMOD International Conference on Management of Data. ACM, 539-550.

[5] Michael Hausenblas and Jacques Nadeau. 2013. Apache Drill: Interactive ad-hoc analysis at scale. Big Data 1, 2 (2013), 100-104.

[6] M. Kornacker et al. 2015. Impala: A modern, open-source SQL engine for Hadoop. In Proceedings of the 7th Biennial Conference on Innovative Data Systems Research.

[7] Thusoo Ashish, Joydeep Sen Sarma, Namit Jain, Zheng Shao, Prasad Chakka, Suresh Anthony, Hao Liu, Pete Wyckoff, and Raghotham Murthy. 2009. Hive: A warehousing solution over a map-reduce framework. Proceedings of the VLDB Endowment 2, 2 (2009), 1626-1629.

[8] Saha Bikas, Hitesh Shah, Siddharth Seth, Gopal Vijayaraghavan, Arun Murthy, and Carlo Curino. 2015. Apache tez: A unifying framework for modeling and building data processing applications. In Proceedings of the 2015 ACM SIGMOD International Conference on Management of Data. ACM, 1357-1369.

[9] Chien-Hsing Wu, Shu-Chen Kao, and Koji Okuhara. 2013. Examination and comparison of conflicting data in granulated datasets: Equal width interval vs. Equal Frequency Interval. Inf. Sci. 239 (2013), 154-164.

[10] Amdahl's law. Retrieved June 22, 2021 from https://en.wikipedia.org/wiki/Amdahl\%27s_law.

[11] Jie Song, HongYan He, Zhi Wang, Ge Yu, and Jean-Marc Pierson. 2018. Modulo based data placement algorithm for energy consumption optimization of MapReduce system. F. Grid Comput 16, 3 (2018), 409-424.

[12] Apache Hadoop. Retrieved June 22, 2021 from https://hadoop.apache.org/.

[13] Huang Shengsheng, Huang Jie, Dai Jinquan, Xie Tao, and Huang. Bo. 2011. The HiBench benchmark suite: Characterization of the MapReduce-based data analysis. New Frontiers in Information and Software as Services. Springer, Berlin.

[14] Owen O’Malley. 2008. Terabyte sort on Apache Hadoop. Retrieved June 22, 2021 from http://sortbenchmark.org/ Yahoo-Hadoop.pdf.

[15] Jie Song Shu Xu, Li Zhang, Claus Pahl, and Ge Yu. 2015. Performance and energy optimization on Terasort algorithm by task self-resizing. Inf. Technol. Control 44, 1 (2015), 30-40.

[16] Floratou Avrilia, Umar Farooq Minhas, and Fatma Özcan. 2014. SQL-on-Hadoop: full circle back to shared-nothing database architectures. Proceedings of the VLDB Endowment 7, 12 (2014), 1295-1306.

[17] Apache Parquet. Retrieved June 22, 2021 from http://parquet.apache.org/.

[18] Requeno José Ignacio, Iñigo Gascón, and José Merseguer. 2018. Towards the performance analysis of Apache tez applications. In Companion of the 2018 ACM/SPEC International Conference on Performance Engineering. ACM, 147-152.

[19] Christian Prokopp. 2014. ORC: An intelligent big data file format for Hadoop and Hive. Retrieved June 22, 2021 from https://www.semantikoz.com/blog/orc-intelligent-big-data-file-format-hadoop-hive/.

[20] Tapdiya Ashish and Daniel Fabbri. 2017. A comparative analysis of state-of-the-art SQL-on-Hadoop systems for interactive analytics. In IEEE International Conference on Big Data (Big Data'17). IEEE, 1349-1356.

[21] Agarwal Sameer, Henry Milner, Ariel Kleiner, Ameet Talwalkar, Michael Jordan, Samuel Madden, Barzan Mozafari, and Ion Stoica. 2014. Knowing when you're wrong: Building fast and reliable approximate query processing systems. In Proceedings of the 2014 ACM SIGMOD International Conference on Management of Data. ACM, 481-492. 
[22] Qarabaqi Bahar and Mirek Riedewald. 2016. Merlin: Exploratory analysis with imprecise queries. IEEE Transactions on Knowledge and Data Engineering 28, 2 (2016), 342-355.

[23] Guagliardo Paolo and Leonid Libkin. 2016. Making SQL queries correct on incomplete databases: A feasibility study. In Proceedings of the 35th ACM SIGMOD-SIGACT-SIGAI Symposium on Principles of Database Systems. ACM, 211-223.

[24] Dimitriadou Kyriaki, Olga Papaemmanouil, and Yanlei Diao. 2014. Explore-by-example: An automatic query steering framework for interactive data exploration. In Proceedings of the 2014 ACM SIGMOD International Conference on Management of Data. ACM, 517-528.

[25] Hina A. Khan, Mohamed A. Sharaf, and Abdullah Albarrak. 2014. DivIDE: Efficient diversification for interactive data exploration. In Proceedings of the 26th International Conference on Scientific and Statistical Database Management. ACM, 15.

[26] Oracle Inc. 2018. Query relaxation. Retrieved June 22, 2021 from https://docs.oracle.com/database/121/CCAPP/GUID7DD2AF6B-88FD-40B7-A522-3F59309D3B35.htm.

[27] Mottin Davide, Alice Marascu, Senjuti Basu Roy, Gautam Das, Themis Palpanas, and Yannis Velegrakis. 2014. IQR: An interactive query relaxation system for the empty-answer problem. In Proceedings of the 2014 ACM SIGMOD International Conference on Management of Data. ACM, 1095-1098.

[28] Kantere Verena, George Orfanoudakis, Anastasios Kementsietsidis, and Timos Sellis. 2015. Query relaxation across heterogeneous data sources. In Proceedings of the 24th ACM International on Conference on Information and Knowledge Management. ACM, 473-482.

[29] Joseph M. Hellerstein, Peter J. Haas, and Helen J. Wang. 1997. Online aggregation. Sigmod Record 26, 2 (1997), 171-182.

[30] Joseph M. Hellerstein, Ron Avnur, Andy Chou, Christian Hidber, Chris Olston, Vijayshankar Raman, Tali Roth, and Peter J. Haas. 1999. Interactive data analysis: The control project. Computer 32, 8 (1999), 51-59.

[31] Joglekar Manas, Hector Garcia-Molina, and Aditya G. Parameswaran. 2017. Interactive data exploration with smart drill-down (extended version). IEEE Transactions on Knowledge \& Data Engineering 1 (2017), 1-1.

[32] Miao Xiaoye, Yunjun Gao, Su Guo, and Wanqi Liu. 2017. Incomplete data management: A survey. Frontiers of Computer Science (2017), 1-22.

[33] Antova Lyublena, Christoph Koch, and Dan Olteanu. 2007. From complete to incomplete information and back. In Proceedings of the 2007 ACM SIGMOD International Conference on Management of Data. ACM, 713-724.

[34] Miao Xiaoye, Yunjun Gao, Baihua Zheng, Gang Chen, and Huiyong Cui. 2016. Top-k dominating queries on incomplete data. IEEE Transactions on Knowledge and Data Engineering 28, 1 (2016), 252-266.

[35] Miao Xiaoye, Yunjun Gao, Gang Chen, Baihua Zheng, and Huiyong Cui. 2016. Processing incomplete k nearest neighbor search. IEEE Transactions on Fuzzy Systems 24, 6 (2016), 1349-1363.

[36] Cheng Wei, Xiaoming Jin, Jian-Tao Sun, Xuemin Lin, Xiang Zhang, and Wei Wang. 2014. Searching dimension incomplete databases. IEEE Transactions on Knowledge and Data Engineering 26, 3 (2014), 725-738.

[37] Robert B. Miller. 1968. Response time in man-computer conversational transactions. In Proceedings of the December 9-11, 1968, Fall foint Computer Conference part I. ACM, 267-277.

[38] Ron Kohavi. 1995. A study of cross-validation and bootstrap for accuracy estimation and model selection. Ijcai 14, 2 (1995), 1137-1145.

[39] Park Yongjoo, Michael Cafarella, and Barzan Mozafari. 2016. Visualization-aware sampling for very large databases. In IEEE 32nd International Conference on Data Engineering (ICDE'16). IEEE, 755-766.

[40] Kim Albert, Eric Blais, Aditya Parameswaran, Piotr Indyk, Sam Madden, and Ronitt Rubinfeld. 2015. Rapid sampling for visualizations with ordering guarantees. Proceedings of the VLDB Endowment 8, 5 (2015), 521-532.

[41] Armbrust Michael, Bill Chambers, and Matei Zaharia. 2017. Databricks delta: A unified data management system for real-time big data. Retrieved June 30, 2021 from https://databricks.com/blog/2017/10/25/databricks-delta-a-unifiedmanagement-system-for-real-time-big-data.html.

[42] Ramnarayan Jags, Barzan Mozafari, Sumedh Wale, Sudhir Menon, Neeraj Kumar, Hemant Bhanawat, Soubhik Chakraborty, Yogesh Mahajan, Rishitesh Mishra, and Kishor Bachhav. 2016. SnappyData: A hybrid transactional analytical store built on spark. In Proceedings of the 2016 International Conference on Management of Data. ACM, 2153-2156.

[43] Presto: Distributed SQL query engine for big data. Retrieved June 22, 2021 from https://prestodb.io/.

[44] Dalvi Nilesh and Dan Suciu. 2007. Efficient query evaluation on probabilistic databases. The VLDB fournal-The International fournal on Very Large Data Bases 16, 4 (2007), 523-544.

[45] Zeng Kai, Shi Gao, Jiaqi Gu, Barzan Mozafari, and Carlo Zaniolo. 2014. ABS: A system for scalable approximate queries with accuracy guarantees. In Proceedings of the 2014 ACM SIGMOD International Conference on Management of Data. ACM, 1067-1070.

[46] Minos N. Garofalakis and Phillip B. Gibbons. 2001. Approximate query processing: Taming the terabytes. In VLDB. 343-352. 
[47] Cormode Graham, Minos Garofalakis, Peter J. Haas, and Chris Jermaine. 2011. Synopses for massive data: Samples, histograms, wavelets, sketches. Foundations and Trends in Databases 4, 1-3 (2011), 1-294.

[48] Chaudhuri Surajit, Bolin Ding, and Srikanth Kandula. 2017. Approximate query processing: No silver bullet. In Proceedings of the 2017 ACM International Conference on Management of Data. ACM, 511-519.

[49] Wang Yijie, Xiaoyong Li, Xiaoling Li, and Yuan Wang. 2013. A survey of queries over uncertain data. Knowledge and Information Systems 37, 3 (2013), 485-530.

[50] Sarma Anish Das, Omar Benjelloun, Alon Halevy, and Jennifer Widom. 2006. Working models for uncertain data. In Proceedings of the 22nd International Conference on Data Engineering (ICDE'06). IEEE, 7-7.

[51] Mohamed A. Soliman, Ihab F. Ilyas, and Kevin Chen-Chuan Chang. 2008. Probabilistic top-k and ranking-aggregate queries. ACM Transactions on Database Systems 33, 3 (2008), 13.

[52] Cheng Reynold, Dmitri V. Kalashnikov, and Sunil Prabhakar. 2007. Evaluation of probabilistic queries over imprecise data in constantly-evolving environments. Information Systems 32, 1 (2007), 104-130.

[53] Cheng Reynold, Dmitri V. Kalashnikov, and Sunil Prabhakar. 2004. Querying imprecise data in moving object environments. IEEE Transactions on Knowledge and Data Engineering 16, 9 (2004), 1112-1127.

Received September 2020; revised January 2021; accepted May 2021 